Pacific Journal of Mathematics

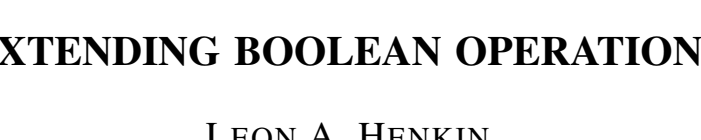




\title{
EXTENDING BOOLEAN OPERATIONS
}

\author{
LEON HENKIN
}

The Stone representation theory provides a canonical method whereby each Boolean algebra $A$ can be embedded isomorphically in a complete, atomistic Boolean algebra $A^{*}$. Jonsson and Tarski have shown how each additive operation (of any number of places) on $A$ can be extended canonically to a completely additive operation on $A^{*}$, in such a way that whenever an equation involving given additive operations holds identically in $A$, the corresponding equation involving the canonical extensions of those operations will hold identically in $A^{*}$. In this paper we present a generalization of this result.

In order to obtain the stated result involving arbitrary equations, it is necessary to consider the smallest set $\Phi$ of operations on $A$, such that $\Phi$ contains all additive operations on $A$ and is closed under composition of operations. The fact that the elements of $\Phi$ are not in general additive complicates the proof of the Jonsson-Tarski theorem. As a by-product of our work we obtain, in the case of a complete Boolean algebra $A$, a characterization of $\Phi$ in terms of equational identities which generalize the notion of additivity.

In $\S 1$ we present three results, needed in the sequel, concerning finite intersections of ultrafilters in an arbitrary Boolean algebra $A$. In $\S 2$ we introduce various sets of operations (on an arbitrary Boolean algebra) with which we shall be concerned, and we discuss the relations among them. In $\S 3$ we associate with each operation $f$ on $A$ a canonical extension $f^{*}$ on $A^{*}$, and prove our basic results about them. Finally, in $\S 4$, we indicate several ways in which the results of $\S 3$ can be strengthened.

1. Thoughout this section we consider an arbitrary Boolean algebra

$$
\mathfrak{U}=\langle A,+, \cdot,-, 0,1\rangle .
$$

We use the term filter to denote what is sometimes called a dualideal, i.e., a nonempty set $X \subseteq A$ which is closed under - and is such that whenever $x \in X$ and $x \leqq y$ then also $y \in X$. We use the term ultrafilter to denote maximal proper filters of $\mathfrak{A}$; these are filters $X$ such that for every $x \in A$ we have either $x \in X$ or $-x \in X$, but not both.

THEOREM 1.1. Let $n$ be a positive integer and suppose that $Y$, $X_{0}, \cdots, X_{n-1}$ are ultrafilters of $\mathfrak{A}$. If $Y \supseteqq \bigcap_{i<n} X_{i}$ then $Y=X_{i}$ for some $i<n$. 
Proof. Assume that $Y \supseteqq \bigcap_{i<n} X_{i}$ and that for each $i<n-1$ we have $Y \neq X_{i}$. We shall show that we must have $Y=X_{n-1}$.

From our second assumption we conclude that for each $i<n-1$ there is some $y_{i} \in Y$ such that $-y_{i} \in X_{i}$. Putting $y=\Pi_{i<n-1} y_{i}$ we conclude that $y \in Y$ and $-y \in \bigcap_{i<n-1} X_{i}$. From the latter, we see that for every $x \in X_{n-1}$ we have

$$
(-y+x) \in \bigcap_{i<n} X_{i},
$$

and hence by our assumption that $Y \supseteqq \bigcap_{i<n} X_{i}$ we have $(-y+x) \in Y$ for every $x \in X_{n-1}$. But $y \in Y$ and $Y$ is a filter; hence $x \in Y$ for each $x \in X_{n-1}$, that is, $X_{n-1} \subseteq Y$. Since $Y$ and $X_{n-1}$ are ultrafilters, this shows that $Y=X_{n-1}$, as claimed.

In order to formulate our second result about finite intersections of ultrafilters, we introduce notation which will be used heavily in the sequel. If $X$ is any set, we denote the cardinal number of $X$ by $|X|$.

Definition 1.2. Suppose $m$ is a positive integer and $X \subseteq A$. We set

$$
\sigma_{m} X=\left\{y \in A: y=x_{0}+\cdots+x_{m-1} \text { for some } x_{0}, \cdots, x_{m-1} \in X\right\} \text {. }
$$

THEOREM 1.3. Let $Y$ be a proper filter of $\mathfrak{A}$ and $m$ a positive integer. In order that there exist ultrafilters $X_{0}, \cdots, X_{m-1}$ of $\mathfrak{A}$ such that $Y=\bigcap_{j<m} X_{j}$, it is necessary and sufficient that whenever $Z \subseteq A$, $|Z| \leqq m+1$, and $\Sigma Z=1$, we have $Y \cap \sigma_{m} Z \neq 0$.

Proof. To prove necessity, assume that $Y=\bigcap_{j<m} X_{j}$ for some ultrafilters $X_{0}, \cdots, X_{m-1}$, and consider any $Z \cong A$ such that $|Z| \leqq m+1$ and $\Sigma Z=1$. From this last equation we see that for each $j<m$ there is some $z_{j} \in Z$ such that $z_{j} \in X_{j}$. But then $\bigcap_{j<m} X_{j} \cap \sigma_{m} Z$ is nonempty as claimed, since it contains the element $z_{0}+\cdots+z_{m-1}$.

To prove sufficiency, recall that every proper filter $Y$ can be extended to an ultrafilter and is the intersection of all such ultrafilters. Assume, therefore, that there exist $m+1$ distinct ultrafilters $X_{0}, \cdots$, $X_{m}$ such that $Y \subseteq \bigcap_{j<m+1} X_{j}$; we shall show that there is some $Z \cong A$ such that $|Z|=m+1, \Sigma Z=1$, and $Y \cap \sigma_{m} z=0$.

Since $X_{0}, \cdots, X_{m}$ are distinct ultrafilters we can find elements $x_{0}, \cdots, x_{m-1}$, with $x_{j} \in X_{j}$ for each $j<m$, such that $x_{j} \notin X_{i}$, and hence $-x_{j} \in X_{i}$, whenever $j \neq i, i=0, \cdots, m$. Putting $x_{m}=\Pi_{j<m}-x_{j}$ we infer that $x_{m} \in X_{m}$ while $-x_{m} \in X_{j}$ for each $j<m$. The elements $x_{0}, \cdots, x_{m}$ are distinct (indeed, they are pairwise disjoint, nonzero elements of $A$ ), so that if we put $Z=\left\{x_{0}, \cdots, x_{m}\right\}$ we have $|z|=m+1$. Also $\Sigma Z=1$. To see $Y \cap \sigma_{m} Z=0$ assume, on the contrary, that there is some $y \in Y \cap \sigma_{m} Z$. Then by 1.2 , there must be elements $z_{0}, \cdots, z_{m-1} \in Z$ with $y=z_{0}+\cdots+z_{m-1}$. We can, therefore, find an 
element $x_{j} \in Z, j<m+1$, such that $y \leqq-x_{j}$ and hence $y \notin X_{j}$. But since $y \in Y$, this contradicts our hypothesis that $Y \subseteq \bigcap_{j<m+1} X_{j}$. This contradiction, arising from the assumption $Y \cap \sigma_{m} Z \neq 0$, completes our proof.

We conclude this section with another characterization of finite intersections of ultrafilters.

THEOREM 1.4. Let $W$ be a filter of $\mathfrak{A}, \quad p$ a positive integer. Then the following conditions are equivalent:

(i) Whenever $Z \subseteq A, Z$ is finite, and $\Sigma Z \in W$, then for some $t \in \sigma_{p} Z$ we have $t \in W$.

(ii) There is some set $G$ of ultrafilters of $\mathfrak{A}$ such that $|G| \leqq p$ and $W=\bigcap G$.

Proof. First assume that (i) holds. In case $0 \in W$ so that $W=A$, we can show that (ii) is satisfied by taking $G$ to be empty. If $0 \notin W$, so that $W$ is a proper filter, we can apply 1.3 to obtain (ii), since whenever $\Sigma Z=1$ we have $\Sigma Z \in W$ and hence $W \cap \sigma_{p} Z \neq 0$ by (i).

Conversely, assume that (ii) holds. If $G$ is empty so that $W=A$, then (i) holds trivially. In the contrary case, say $G=\left\{X_{0}, \cdots, X_{p-1}\right\}$ where each $X_{i}$ is an ultrafilter of $\mathfrak{A}$. Now take any finite $Z \subseteq A$ such that $\Sigma Z \in W$. Then by (ii) we have $\Sigma Z \in X_{i}$ for each ultrafilter $X_{i} \in G$. Hence for each $i<p$ there is some $Z_{i} \in z$ such that $Z_{i} \in X_{i}$. But then

$$
z_{0}+\cdots+z_{p-1} \in \bigcap_{i<p} X_{i} .
$$

Putting $t=\Sigma_{i<p} z_{i}$ we thus have $t \in \sigma_{p} Z$ and $t \in W$ by (ii). This proves (i), and completes the proof that (i) and (ii) are equivalent.

2. We again consider an arbitrary Boolean algebra

$$
\mathfrak{U}=\langle A,+, \cdot,-, 0,1\rangle,
$$

and we now define certain sets of operations on $A$ with which we shall be concerned in $\S 3$ below. For any positive integer $n$ we denote by ${ }^{n} A$ the $n$-fold Cartesian power of $A$, and for any $x \in{ }^{n} A$ and each $i<n$ we let $x_{i}$ be the $i$ th projection of $x$ on $A$. An $n$-place operation on $A$ is simply a function from ${ }^{n} A$ to $A$. In the following definition we use the notations $x+y$ and $x \leqq y$ where $x, y \in{ }^{n} A$. The operation + and the relation $\leqq$ are here understood to be those of the Boolean algebra ${ }^{n} \mathfrak{A}$, so that $(x+y)_{j}=x_{j}+y_{j}$ for each $j<n$, and $x \leqq y$ if and only if $\left(x_{j} \leqq y_{j}\right.$ for every $\left.j<n\right)$. Also, if $j, n$ are positive integers such that $j<n$, and if $x, y \in{ }^{n} A$, we use the notation $x={ }_{j} y$ to mean that $x_{i}=y_{i}$ for all $i \neq j, i<n$. 
DEFINITION 2.1. Let $n$ be a positive integer and $f$ an $n$-place operation on $A$. We say that $f$ is

(i) monotonic if, whenever $x, y \in{ }^{n} A$ and $x \leqq y$, we have $f x \leqq f y$;

(ii) $p$-additive (where $p$ is any positive integer) if, whenever $X \leqq{ }^{n} A,|X| \leqq p+1$, and there is a $j<n$ such that $x={ }_{j} y$ for all $x, y \in X$, we have

$$
f(\Sigma X)=\Sigma\left\{f z: z \in \sigma_{p} X\right\} ;
$$

(iii) completely $p$-additive if, whenever $X \subseteq{ }^{n} A$, and there is a $j<n$ such that $x={ }_{j} y$ for all $x, y \in X$, and $\Sigma X$ exists in ${ }^{n} \mathfrak{A}$, then also $\Sigma\left\{f z: z \in \sigma_{p} X\right\}$ exists and equals $f(\Sigma X)$.

For each positive integer $\rho$ we let $\Phi_{p}(\mathfrak{A})$ (or simply $\Phi_{p}$ ) be the set of all $p$-additive operations on $\mathfrak{A}$, and we set $\Phi_{\omega}=\bigcup_{p} \Phi_{p}$, the union being taken over all positive integers $p$. Similarly, we let $\Phi_{p}^{c}(\mathfrak{Q})$ be the set of all completely $p$-additive operations on $\mathfrak{U}$, and we set $\Phi_{\omega}^{c}(\mathfrak{Q})=\bigcup_{p} \Phi_{p}^{c}(\mathfrak{H})$.

REMARK 2.2. For any positive integers $n$ and $p$ one can easily derive from Definitions 2.1 and 1.2 a set of $n$ equational identities in $n+p$ variables characterizing those $n$-place operations which are in $\Phi_{p}$. For example, a 3-place operation $f$ on a Boolean algebra $\mathfrak{A}$ will be in $\Phi_{2}(\mathfrak{U})$ if and only if the following equations hold identically in $\mathfrak{U}$ :

$$
\begin{aligned}
f\left(x_{0}+x_{1}+x_{2}, y, z\right)= & f\left(x_{0}+x_{1}, y, z\right) \\
& +f\left(x_{0}+x_{2}, y, z\right)+f\left(x_{1}+x_{2}, y, z\right) \\
f\left(x, y_{0}+y_{1}+y_{2}, z\right)= & f\left(x, y_{0}+y_{1}, z\right) \\
& +f\left(x, y_{0}+y_{2}, z\right)+f\left(x, y_{1}+y_{2}, z\right) \\
f\left(x, y, z_{0}+z_{1}+z_{2}\right)= & f\left(x, y, z_{0}+z_{1}\right) \\
& +f\left(x, y, z_{0}+z_{2}\right)+f\left(x, y, z_{1}+z_{2}\right) .
\end{aligned}
$$

From such an equational characterization of $\Phi_{1}$ one sees that the 1-additive operations are precisely those operations called additive by Jonsson and Tarski in [2]. Obviously for each positive integer $p$ we have $\Phi_{p}^{c} \subseteq \Phi_{p}$ and so $\Phi_{\omega}^{c} \subseteq \Phi_{\omega}$. The following theorems give other relations among the sets defined in 2.1, and some basic properties of these sets which will be useful in the sequel.

THEOREM 2.3. If $f \in \Phi_{\omega}$ then $f$ is monotonic.

Proof. By 2.1 it suffices to show, for each positive integer $p$, that every element of $\Phi_{p}$ is monotonic. Suppose, therefore, that $f$ is an $n$-place operation on $A$ with $f \in \Phi_{p}$, and consider any $x, y \in{ }^{n} A$ such 
that $x \leqq y$. We must show that $f x \leqq f y$.

Choose $x^{(0)}, \cdots, x^{(n-1)} \in{ }^{n} A$ so that $x^{(0)}=x$, and for each $i=0, \cdots$, $n-2$ we have $x^{(i+1)}={ }_{i+1} x^{(i)}$ and $x_{i+1}^{(i+1)}=y_{i+1}$. Clearly, then, we will have $x^{(i)} \leqq x^{(i+1)}$ for each $i=0, \cdots, n-2$, and $x^{(n-1)}=y$. By transitivity of $\leqq$ it suffices for us to show that $f x^{(i)} \leqq f x^{(i+1)}$ for all $i<n-1$.

Fixing an arbitrary $i<n-1$, take $X=\left\{x^{(i)}, x^{(i+1)}\right\}$. Then, since $f \in \Phi_{p}$, we can conclude (by 2.1 and 1.2) that

$$
f\left(x^{(i)}+x^{(i+1)}\right)=f x^{(i)}+f x^{(i+1)}+f\left(x^{(i)}+x^{(i+1)}\right)
$$

- the last term on the right being omitted in case $p=1$. Since $x^{(i)} \leqq$ $x^{(i+1)}$ we thus get $f x^{(i+1)}=f x^{(i)}+f x^{(i+1)}$, which gives $f x^{(i)} \leqq f x^{(i+1)}$ as desired.

LEMMA 2.4. Let $f$ be an n-place operation on $A$. In order that $f \in \Phi_{p}$ it is necessary and sufficient that for every finite $X \subseteq{ }^{n} A$ such that for some $j<n$ we have $x={ }_{j} y$ for all $x, y \in X$,

$$
f(\Sigma X)=\Sigma\left\{f y: y \in \sigma_{p} X\right\} .
$$

Proof. Sufficiency is immediate from 2.1. Necessity is proved by induction on $|X|$, as follows.

Assume that $f \in \Phi_{p}$. If $|X| \leqq p+1$ we see that equation $\left(^{*}\right)$ will hold directly from 2.1. Consider, therefore, any $q \geqq p+1$. Let us make the induction hypothesis that the equation. $\left({ }^{*}\right)$ holds whenever $|X| \leqq q$, and consider an arbitrary set $Y=\left\{y^{(0)}, \cdots, y^{(q)}\right\}$ containing $q+1$ distinct elements of ${ }^{n} A$ such that for some $j<n$ we have $y^{(i)}={ }_{j} y^{(k)}$ for all $i, k \leqq q$.

Put $x^{(i)}=y^{(i)}$ for $i=0, \cdots, q-2$ and $x^{(q-1)}=y^{(q-1)}+y^{(q)}$. Setting $X=\left\{x^{(0)}, \cdots, x^{(q-1)}\right\}$ we obviously have $x^{(i)}={ }_{j} x^{(k)}$ for all $i, k<q$, and so we can apply our induction hypothesis to obtain

$$
f(\Sigma X)=\Sigma\left\{f z: z \in \sigma_{p} X\right\}
$$

Also, we clearly have

$$
\Sigma X=\Sigma Y \text {. }
$$

Now consider any $z \in \sigma_{p} X$. By 1.2 and definition of $X$ we clearly have $z=\Sigma Z$ for some $Z \leqq Y$ such that $|Z| \leqq p+1$. Hence, since $f \in \Phi_{p}$, we get

$$
\begin{aligned}
f z & =\Sigma\left\{f t: t \in \sigma_{p} Z\right\} \\
& \leqq \Sigma\left\{f t: t \in \sigma_{p} Y\right\} .
\end{aligned}
$$

Since this holds for each $z \in \sigma_{p} X$, we thus get, 


$$
\Sigma\left\{f z: z \in \sigma_{p} X\right\} \leqq \Sigma\left\{f t: t \in \sigma_{p} Y\right\}
$$

which, with (1) and (2) gives

$$
f(\Sigma Y) \leqq \Sigma\left\{f t: t \in \sigma_{p} Y\right\} .
$$

The opposite inequality follows from the monotonicity of $f$. Hence in (3) we may replace $\leqq$ by $=$, showing that the equation $\left(^{*}\right)$ holds for $Y$. This completes our proof by mathematical induction.

CoROLlaRY 2.5. If $p \leqq q$ then $\Phi_{p} \subseteq \Phi_{q}$.

Proof. Suppose $p \leqq q$ and let $f \in \Phi_{p}$; say $f$ is an $n$-ary operation on $A$. Consider any $X \cong{ }^{n} A$ such that $|X| \leqq q+1$, and such that for some $j<n$ we have $x={ }_{j} y$ for all $x, y \in X$. By 2.4 we have

$$
f(\Sigma X)=\Sigma\left\{f y: y \in \sigma_{p} X\right\} .
$$

Since, by 1.2 , we have $\sigma_{p} X \subseteq \sigma_{q} X$, this gives

$$
f(\Sigma X) \leqq \Sigma\left\{f y: y \in \sigma_{q} X\right\} .
$$

The converse inequality follows by 2.3 , which gives $f \in \Phi_{q}$ by 2.1 . This proves 2.5 .

REMARK 2.6. In general we have $\Phi_{p}(\mathfrak{U} \varsubsetneqq) \Phi_{q}(\mathfrak{U})$ when $p<q$. For example, let $A$ be the set of all binary relations on $\omega$ (the set of all natural numbers), and consider the Boolean algebra

$$
\mathfrak{U}=\left\langle A, \cup, \cap, \sim, \varnothing,{ }^{2} \omega\right\rangle \text {. }
$$

To obtain an operation in $\Phi_{2}$ which is not in $\Phi_{1}$, consider the 1place operation $f$ on $A$ such that $f x=x ; x$ for every $x \in A$. (Here $x ; x$ is the relative product of the relation $x$ with itself, so that for any $i, j \in \omega$ we have $\langle i, j\rangle \in f x$ if and only if there is some $k \in \omega$ such that $\langle i, k\rangle \in x$ and $\langle k, j\rangle \in x$.)

To see that $f \notin \Phi_{1}$, consider the relations $x=\{\langle 0,1\rangle\}$ and $y=\{\langle 1,2\rangle\}$. Clearly $f x=f y=\varnothing$, but $f(x \cup y)=\{\langle 0,2\rangle\}$; hence $f(x \cup y) \neq f x \cup f y$. On the other hand it is easy to see that for any relations $z_{0}, z_{1}, z_{2} \in A$ we have

$$
f\left(z_{0} \cup z_{1} \cup z_{2}\right)=f\left(z_{0} \cup z_{1}\right) \cup f\left(z_{0} \cup z_{2}\right) \cup f\left(z_{1} \cup z_{2}\right),
$$

so that $f \in \Phi_{2}$.

This idea is easily elaborated to obtain a 1-place operation $f_{p} \in\left(\Phi_{p+1} \sim \Phi_{p}\right)$ for each $p=1,2, \cdots$. Simply take $f_{1}$ to be the operation $f$ above, and set $f_{p+1} x=\left(f_{p} x\right) ; x$ for each $x \in A$. 
THEOREM 2.7. Let $g$ be an $(n+1)$-place operation on $A$ with $g \in \Phi_{p}$ (resp., $g \in \Phi_{p}^{c}$ ). Let $t \in A$, and let $f$ be the n-place operation on $A$ such that, for each $z \in{ }^{n} A, f z=g\left(z_{0}, \cdots, z_{n-1}, t\right)$. Then $f \in \Phi_{p}$ (resp., $\left.f \in \Phi_{p}^{c}\right)$.

Proof. Obvious.

The following theorem generalizes the distributive law for additive operations.

THEOREM 2.8. Let $f$ be an n-place operation on A. For each $X \subseteq{ }^{n} A$ and $j<n$ let $X_{j}=\left\{x_{j}: x \in X\right\}$, and set

$$
X^{+}=X_{0} \times X_{1} \times \cdots \times X_{n-1} .
$$

In order that $f \in \Phi_{p}$ (resp., $f \in \Phi_{p}^{c}$ ) it is necessary and sufficient that for every finite $X \subseteq{ }^{n} A$ (resp., every $X \subseteq{ }^{n} A$ such that $\Sigma X$ exists in $\left.{ }^{n} \mathfrak{U}\right)$ we have

$$
f(\Sigma X)=\Sigma\left\{f t: t \in \sigma_{p}\left(X^{+}\right)\right\}
$$

Proof. The sufficiency of $\left(^{*}\right)$ is immediate from the fact that whenever $X \subseteq{ }^{n} A$, and for some $j<n$ we have $x={ }_{j} y$ for all $x, y \in X$, then $X^{+}=X$. Necessity is proved by induction on $n$. In case $n=1$ we see that $\left({ }^{*}\right)$ follows directly from 2.4 (resp., 2.1 (iii)), since $X^{+}=X$ for any $X \subseteq A$.

Now make the induction hypothesis that $(*)$ holds for any $n$-place speration $f \in \Phi_{p}$ (resp., $\left.f \in \Phi_{p}^{c}\right)$. Consider any $(n+1)$-place $g \in \Phi_{p}$ (resp., $g \in \Phi_{p}^{c}$ ), and any $X \subseteq{ }^{(n+1)} A$ such that $X$ is finite (resp., such that $\Sigma X$ exists in $\left.{ }^{(n+1)} \mathfrak{A}\right)$.

For each $x \in \in^{(n+1)} A$ let $y^{(x)} \in{ }^{(n+1)} A$ be such that $y_{j}^{(x)}=(\Sigma X)_{j}$ for each $j<n$ and $y_{n}^{(x)}=x_{n}$. Let $Y=\left\{y^{(x)}: x \in X\right\}$. Clearly $\Sigma Y=\Sigma X$ and $y={ }_{n} z$ for all $y, z \in Y$; hence our hypothesis on $g$ yields

$$
g(\Sigma X)=\Sigma\left\{g u: u \in \sigma_{p} Y\right\} .
$$

By definition of $X^{+}$and $Y$ we see that for any $t \in \sigma_{p}\left(X^{+}\right)$there is some $u \in \sigma_{p} Y$ such that $u \geqq t$. Hence from (1) and the monotonicity of $g$ we obtain

$$
g(\Sigma X) \geqq \Sigma\left\{g t: t \in \sigma_{p}\left(X^{+}\right)\right\}
$$

Next consider any $u \in \sigma_{p} Y$. By definition of $Y$ we see that

$$
u=y^{(t)} \text { for some } t \in \sigma_{p} X \text {. }
$$

Choosing and fixing such a $t$, we consider the $n$-place operation $f$ such 
that for any $z \in{ }^{n} A$ we have $f z=g\left(z_{0}, \cdots, z_{n-1}, t_{n}\right)$. By 2.7, $f \in \Phi_{p}$ (resp., $f \in \Phi_{p}^{c}$ ).

Now for each $w \in{ }^{(n+1)} A$ let $w^{\prime} \in{ }^{n} A$ be such that $w_{j}^{\prime}=w_{j}$ for all $j<n$, and for each $W \cong{ }^{(n+1)} A$ let $W^{\prime}=\left\{w^{\prime}: w \in W\right\}$. Since $\Sigma X$ exists in ${ }^{(n+1)} A$, clearly $\Sigma\left(X^{\prime}\right)$ exists in ${ }^{n} A$ and $\Sigma\left(X^{\prime}\right)=(\Sigma X)^{\prime}$. Also $\left(X^{\prime}\right)^{+}=$ $\left(X^{+}\right)^{\prime}$. Hence we can apply our induction hypothesis to the $n$-place operation $f$, obtaining

$$
f\left((\Sigma X)^{\prime}\right)=\Sigma\left\{f v: v \in \sigma_{p}\left(X^{+^{\prime}}\right)\right\} .
$$

But by definition of $f$ we have

$$
\begin{aligned}
f\left((\Sigma X)^{\prime}\right) & =g\left((\Sigma X)_{0}, \cdots,(\Sigma X)_{n-1}, t_{n}\right) \\
& =g u \text { by (3) and definition of } y^{(t)} .
\end{aligned}
$$

Hence from (4) we get

$$
g u=\Sigma\left\{f v: v \in \sigma_{p}\left(X^{+\prime}\right)\right\} .
$$

Finally, for any $v \in \sigma_{p}\left(X^{+\prime}\right)$ we have $f v=g w$ for some $w \in \sigma_{p}\left(X^{+}\right)$, as we see from the definitions of $f$ and $X^{+}$by taking $w_{j}=v_{j}$ for each $j<n$ and $w_{n}=t_{n}$, and recalling that $t \in \sigma_{p} X$ (cf. (3)). Hence (5) yields

$$
g u \leqq \Sigma\left\{g w: w \in \sigma_{p}\left(X^{+}\right)\right\} \text {. }
$$

Since $u$ was chosen as an arbitrary element of $\sigma_{p} Y$, we can combine (1) with (6) to obtain

$$
g(\Sigma X) \leqq \Sigma\left\{g w: w \in \sigma_{p}\left(X^{+}\right)\right\}
$$

Putting (2) and (7) together, we see that the equation $\left(^{*}\right)$ of Theorem 2.8 holds for the $(n+1)$-place operation $g$, completing our proof by mathematical induction.

CoRollary 2.9. Let $f$ be an n-place operation on $A$ with $f \in \Phi_{p}$ (resp., $f \in \Phi_{p}^{c}$ ). Put $q=n \cdot p$. Then for any $X \cong{ }^{n} A$ such that $X$ is finite (resp., such that $\Sigma X$ exists in ${ }^{n} \mathfrak{U}$ ) we have

$$
f(\Sigma X)=\Sigma\left\{f u: u \in \sigma_{q} X\right\} \text {. }
$$

Proof. Let $X^{+}$be obtained from $X$ as in 2.8. It is evident that for any $x \in X^{+}$there is some $y \in \sigma_{n} X$ such that $y \geqq x$, and hence for any $t \in \sigma_{p}\left(X^{+}\right)$there is some $u \in \sigma_{q}(X)$ such that $u \geqq t$, and hence $f u \geqq f t$ by monotonicity of $f$. Applying 2.8 we thus obtain

$$
f(\Sigma X) \leqq \Sigma\left\{f u: u \in \sigma_{q} X\right\} \text {. }
$$

The opposite inequality is again obtained by monotonicity, and 2.9 follows. 
If $f$ is any $m$-place operation and $g_{0}, \cdots, g_{m-1}$ are $n$-place operations on $A$, we can compose them to obtain the operation $f\left[g_{0}, \cdots, g_{m-1}\right]$, i.e., the $n$-place operation $h$ such that $h x=f\left(g_{0} x, \cdots, g_{m-1} x\right)$ for every $x \in{ }^{n} A$. The following theorem shows that the sets $\Phi_{\omega}$ and $\Phi_{\omega}^{c}$ are each closed under composition of operations.

THEOREM 2.10. Let $f$ be an m-place operation, let $g_{0}, \cdots, g_{m-1}$ be n-place operations, and put $h=f\left[g_{0}, \cdots, g_{m-1}\right]$. Assume that $f \in \Phi_{p}$ (resp., $f \in \Phi_{p}^{c}$ ), that $g_{i} \in \Phi_{q}$ (resp., $g_{i} \in \Phi_{p}^{c}$ ) for each $i<m$, and put $r=m \cdot p \cdot q$. Then we have $h \in \Phi_{r}$ (resp., $h \in \Phi_{r}^{c}$ ).

Proof. By definition of $h$ we have $h x=f\left(g_{0} x, \cdots, g_{m-1} x\right)$ for each $x \in{ }^{n} A$. Since $f, g_{0}, \cdots, g_{m-1}$ are all monotonic, it follows that

$h$ is monotonic.

Now consider any $X \subseteq{ }^{n} A$ and $j<n$ such that $x={ }_{j} y$ for all $x, y \in X$; assume $X$ is finite (resp., $\Sigma X$ exists in ${ }^{n} \mathfrak{Q}$ ). For any $i<n$ we obtain, from our hypothesis on $g_{i}$ and 2.4 ,

$$
g_{i}(\Sigma X)=\Sigma\left\{g_{i} t: t \in \sigma_{q} X\right\} \text {. }
$$

Now for each $i<m$ set $Y_{i}=\left\{g_{i} t: t \in \sigma_{q} X\right\}$, and put $Y=Y_{0} \times \cdots \times$ $X_{m-1}$. From (2) and the definition of $h$ we have

$$
\begin{aligned}
h(\Sigma X) & =f\left(\Sigma Y_{0}, \cdots, \Sigma Y_{m-1}\right) \\
& =f(\Sigma Y) \text { by definition of } Y .
\end{aligned}
$$

If we obtain $Y^{+}$from $Y$ in the way described in 2.8 , we see from the definition of $Y$ that in fact $Y^{+}=Y$. Hence by 2.8 and (3) we obtain

$$
h(\Sigma X)=\Sigma\left\{f u: u \in \sigma_{p} Y\right\} .
$$

By the monotonicity of $g_{0}, \cdots, g_{m-1}$ and the definition of $Y$ we see that for each $y \in Y$ we can find some $v \in \sigma_{m \cdot q} X$ such that $y_{i}<g_{i} v$ for every $i<m$. Hence, by the monotonicity of $f$ and $g_{0}, \cdots, g_{m-1}$ we see that for every $u \in \sigma_{p} Y$ we can find some $w \in \sigma_{p \cdot m \cdot q} X$ such that

$$
f u \leqq f\left(g_{0} w, \cdots, g_{m-1} w\right)=h w .
$$

Since $r=m \cdot p \cdot q$ we thus have: For every $u \in \sigma_{p} Y$ there is some $w \in \sigma_{r} X$ such that $f u \leqq h w$. Hence from (4) we get

$$
\begin{aligned}
\text { Whenever } & z \geqq h w \text { for all } w \in \sigma_{r} X, \\
\text { we have } & z \geqq h(\Sigma X) .
\end{aligned}
$$

On the other hand we also have

$$
h(\Sigma X) \geqq h w \text { for all } w \in \sigma_{r} X
$$


by (1). Combining (5) and (6) gives

$$
h(\Sigma X)=\Sigma\left\{h w: w \in \sigma_{r} X\right\} .
$$

From the way in which $X$ was chosen, this shows that $h \in \Phi_{r}$ (resp., $\left.h \in \Phi_{r}^{c}\right)$, as claimed in 2.10 .

In $\S 3$ we shall show how each $f \in \Phi_{p}(\mathfrak{U})$ can be extended to an $f^{*} \in \Phi_{p}^{c}\left(\mathfrak{U}^{*}\right)$, the algebra $\mathfrak{2}^{*}$ being complete and atomistic. In the following two theorems we find that the class $\Phi_{p}^{c}(\mathfrak{B})$ has a particularly simple character for any Boolean algebra $\mathfrak{B}$ which is complete and atomistic.

THEOREM 2.11. Let $\mathfrak{B}$ be a complete, atomistic algebra, and $B^{\prime}$ the set consisting of 0 and all the atoms of $\mathfrak{B}$. Let $f$ be an n-place operation on $B$, and for each $x \in{ }^{n} B$ let $A^{(x)}=\left\{a \in{ }^{n}\left(B^{\prime}\right): a \leqq x\right\}$. In order that $f \in \Phi_{p}^{c}(\mathfrak{B})$ it is necessary and sufficient that for every $x \in{ }^{n} B$ we have

$$
f x=\Sigma\left\{f b: b \in \sigma_{p}\left(A^{(x)}\right)\right\}
$$

Proof. Let us first assume $f \in \Phi_{p}^{c}(\mathfrak{B})$. To show the necessity of (*), observe first that for any $x \in{ }^{n} B$ we have

$$
x=\Sigma\left(A^{(x)}\right)
$$

by the hypothesis that $B$ is atomistic. Next observe that if we form $A^{(x)+}$ from $A^{(x)}$ by the method described in 2.8 , we find that in fact

$$
A^{(x)+}=A^{(x)} \text {. }
$$

By 2.8 we have

$$
f\left(\Sigma\left(A^{(x)}\right)\right)=\Sigma\left\{f b: b \in \sigma_{p}\left(A^{(x)+}\right)\right\},
$$

which, by (1) and (2), yields $\left({ }^{*}\right)$.

Next let us assume that $f$ satisfies the condition $(*)$, and prove that this is sufficient to yield $f \in \Phi_{p}^{c}(B)$.

We first observe that whenever $x \leqq y$ we have $A^{(x)} \subseteq A^{(y)}$, and hence $\sigma_{p}\left(A^{(x)}\right) \leqq \sigma_{p}\left(A^{(y)}\right)$; and we then get $f x \leqq f y$ from $\left(^{*}\right)$. This shows that

$f$ is monotonic.

Next consider any $X \subseteq{ }^{n} A$. Of course $\Sigma X$ exists in ${ }^{n} \mathfrak{B}$ by our hypothesis that $\mathfrak{B}$ is complete, and by $\left({ }^{*}\right)$ we have

$$
f(\Sigma X)=\Sigma\left\{f b: b \in \sigma_{p}\left(A^{(\Sigma X)}\right)\right\} \text {. }
$$


Let $X^{+}$be obtained from $X$ by the method specified in 2.8. If $a \in A^{(\Sigma X)}$ then for each $j<n$ we have $a_{j}<x_{j}$ for some $x \in X$; hence $a \leqq y$ for some $y \in X^{+}$. It follows that for every $b \in \sigma_{p}\left(A^{(\Sigma X)}\right)$ there is some $t \in \sigma_{p}\left(X^{+}\right)$for which $b \leqq t$, and hence $f b \leqq f t$ by (1). Thus

$$
\Sigma\left\{f b: b \in \sigma_{p}\left(A^{(\Sigma X)}\right)\right\} \leqq \Sigma\left\{f t: t \in \sigma_{p}\left(X^{+}\right)\right\},
$$

from which we get, by (2)

$$
f(\Sigma X) \leqq \Sigma\left\{f t: t \in \sigma_{p}\left(X^{+}\right)\right\} \text {. }
$$

Since for each $y \in X^{+}$we have $y \leqq \Sigma X$, we get $t \leqq \Sigma X$ for each $t \in \sigma_{p}\left(X^{+}\right)$and hence

$$
\Sigma\left\{f t: t \in \sigma_{p}\left(X^{+}\right)\right\} \leqq f(\Sigma X)
$$

by (1). Combining (3) and (4) and using 2.8 we conclude that $f \in \Phi_{p}^{c}(\mathfrak{B})$, as desired.

In the next theorem we show that when $\mathfrak{B}$ is complete and atomistic, each operation in $\Phi_{\omega}^{c}(\mathfrak{B})$ can be obtained by composition from operations in $\Phi_{1}^{c}(\mathfrak{B})$. In formulating this theorem we deal with the projections $h^{(n, s)}$ associated with $B$ : If $n$ and $s$ are positive integers such that $n>s$, then $h^{(n, s)}$ is the $n$-place operation on $B$ such that $h^{(n, s)} x=x_{s}$ for each $x \in{ }^{n} B$. Clearly each $h^{(n, s)} \in \Phi_{1}^{c}(\mathfrak{B})$.

Theorem 2.12. Let $\mathfrak{B}$ be any complete, atomistic Boolean algebra, $n$ and $p$ any positive integers, and $q=n \cdot p$. There exists a sequence $s \in{ }^{q}\{0, \cdots, n-1\}$ such that every $n$-place operation $f \in \Phi_{p}^{c}(\mathfrak{B})$ can be obtained from some q-place operation $g \in \Phi_{1}^{c}(\mathfrak{B})$ by the composition

$$
f=g\left[h^{\left(n, 0^{s}\right)}, \cdots, h^{\left(n, s_{q-1}\right)}\right] .
$$

Proof. Since $q=n \cdot p$, we see that for each $k<q$ there exist unique $i<p$ and $j<n$ such that $k=j \cdot p+i$. Thus we can define $s \in q\{0, \cdots, n-1\}$ by the rule that

$$
s_{j \cdot p+j}=j \quad \text { for all } j<n \text { and } i<p \text {. }
$$

Let $B^{\prime}$ be the set consisting of 0 and all the atoms of $\mathfrak{B}$, and set

$$
\begin{array}{ll}
A^{(x)}=\left\{a \in{ }^{q}\left(B^{\prime}\right): a \leqq x\right\} & \text { for each } x \in{ }^{q} B, \\
C^{(w)}=\left\{b \in{ }^{n}\left(B^{\prime}\right): b \leqq w\right\} & \text { for each } w \in{ }^{n} B .
\end{array}
$$

Let $f$ be any $n$-place operation, $f \in \Phi_{p}^{c}(\mathfrak{B})$. Then define $g$ to be the $q$-place operation on $B$ such that, for any $x \in{ }^{q} B$,

$$
g x=\Sigma\left\{f\left(\Sigma_{i<p} a_{i}, \Sigma_{i<p} a_{p+i}, \cdots, \Sigma_{i<p} a_{(n-1) \cdot p+i}\right): a \in A^{(x)}\right\} .
$$


Let $x \in{ }^{q} B$, and consider any $b \in A^{(x)}$. Clearly by (2) we have $b \in A^{(b)}$, and $b \geqq a$ for any $a \in A^{(b)}$, so that by (3) and the monotonicity of $f$ we get

$$
g b=f\left(\Sigma_{i<p} b_{i}, \Sigma_{i<p} b_{p+i}, \cdots, \Sigma_{i<p} b_{(n-1) \cdot p+i}\right)
$$

for each $b \in A^{(x)}$. Referring again to (3) we infer that

$$
g x=\Sigma\left\{g b: b \in A^{(x)}\right\} \quad \text { for each } x \in{ }^{q} B .
$$

Applying 2.11 to (4), and using our hypothesis that $\mathfrak{B}$ is complete and atomistic, we obtain

$$
g \in \Phi_{1}^{c}(\mathfrak{B}) .
$$

It remains to show that equation $\left(^{*}\right)$ holds. For this purpose consider any $w \in{ }^{n} B$, and let $x \in{ }^{q} B$ be such that

$$
x_{k}=h^{\left(n, s_{k}\right)} w \quad \text { for all } k<q,
$$

so that

$$
g x=\left(g\left[h^{\left(n, s_{0}\right)}, \cdots, h^{\left(n, s_{q-1}\right)}\right]\right) w .
$$

Now for each $a \in A^{(x)}$ let $b^{(a)}$ be the element of ${ }^{n} B$ such that

$$
b_{j}^{(a)}=\Sigma_{i<p} a_{j \cdot p+i} \quad \text { for every } j<n,
$$

so that

$$
g x=\Sigma\left\{f b^{(a)}: a \in A^{(x)}\right\},
$$

by (3). For each $j \leqq n$ and $i<p$ we have $x_{j \cdot p+i}=w_{j}$ by (1) and (6), and so by (2), if $a \in A^{(x)}$ we have $a_{j . p+i} \leqq w$ and $a_{j \cdot p+i} \in B^{\prime}$; but then by (8), we get $b_{j}^{(a)} \leqq w_{j}$ and $b_{j} \in \sigma_{p}\left(B^{\prime}\right)$. This shows that

$$
b^{(a)} \in \sigma_{p}\left(C^{(w)}\right) \quad \text { for every } a \in A^{(x)},
$$

by (2). Combining (9) with (10) we get

$$
g x \leqq \Sigma\left\{f b: b \in \sigma_{p}\left(C^{(w)}\right)\right\} .
$$

To obtain the opposite inequality, consider any $b \in \sigma_{p}\left(C^{(w)}\right)$; say $b=b^{(0)}+\cdots+b^{(p-1)}$, with each $b^{(i)} \in C^{(w)}$. Let $a$ be the element of ${ }^{q} B$ such that

$$
a_{j \cdot p+i}=b_{j}^{(i)} \quad \text { for every } i<p \text { and } j<n .
$$

For $i<p$ and $j<n$ we have $b_{j}^{(i)} \leqq w_{j}$ and $b_{j}^{(i)} \in B^{\prime}$ by (2); hence $a_{j \cdot p+i} \leqq x_{j \cdot p+i} \in B^{\prime}$ by (12), (6), and (1), and so $a \in A^{(x)}$ by (2). Then, since $\Sigma_{i<p} a_{j \cdot p+i}=b_{j}$ for each $j<n$, by (12), we conclude that $f b \leqq g x$ by (3). Since $b$ is an arbitrary element of $\sigma_{p}\left(C^{(w)}\right)$, this shows that 


$$
\Sigma\left\{f b: b \in \sigma_{p}\left(C^{(w)}\right)\right\} \leqq g x .
$$

Now $f \in \Phi_{p}^{c}(\mathfrak{B})$ by hypothesis. Hence we can combine (11) and (13) with 2.11 to conclude that $f w=g x$. Hence, by (7), we have $f w=$ $\left(g\left[h^{\left(n, s_{0}\right)}, \cdots, h^{\left(n, s_{q-1}\right)}\right]\right) w$. Since $w$ is an arbitrary element of ${ }^{n} B$, this establishes equation $\left(^{*}\right)$ of 2.12 and, in view of (5), completes the proof of the theorem.

REMARK 2.13. For every finite Boolean algebra we have $\Phi_{p}^{c}(\mathfrak{A})=$ $\Phi_{p}(A)$ by 2.1 and 2.4. However, in any infinite $\mathfrak{U}$ we have $\Phi_{p}^{c}(\mathfrak{U}) \varsubsetneqq$ $\Phi_{p}(\mathfrak{A})$. For let $X$ be any nonprincipal ultrafilter of $\mathfrak{Y}$ and let $f$ be the 1-place operation such that $f x=1$ if $x \in X$ and $f x=0$ if $x \notin X$ for every $x \in A$. Obviously $f \in \Phi_{1}(\mathfrak{H})$, and hence $f \in \Phi_{p}(\mathfrak{H})$ for every $p$ by 2.5. On the other hand for $Y=\{-x: x \in X\}$ we have $\Sigma Y=1$ (since $X$ is nonprincipal), so $f(\Sigma Y)=1$; but $f y=0$ for every $y \in Y$. This shows that $f \notin \Phi_{p}^{c}(\mathfrak{I})$.

From the fact that in general $\Phi_{p}^{c} \neq \Phi_{p}$, we see that we cannot strengthen 2.12 to say that every $f \in \Phi_{p}(\mathfrak{B})$ can be obtained from some $g \in \Phi_{1}^{c}(\mathfrak{B})$ by equation $\left(^{*}\right)$. For whenever $f$ is 1-place and $g \in \Phi_{1}^{c}(\mathfrak{B})$ we have $g\left[h^{\left(n, s_{0}\right)}, \cdots, h^{\left(n, s_{q-1}\right)}\right] \in \Phi_{p}^{c}(\mathfrak{B})$ by 2.10 . The question whether every $f \in \Phi_{p}$ can be obtained from some $g \in \Phi_{1}(\mathfrak{B})$ by $(*)$, involves the Stone extension algebra $\mathfrak{B}^{*}$, and so will be deferred until $\S 3$.

We conclude this section with a lemma about arbitrary $f \in \Phi_{p}(\mathfrak{Z})$, which will be used to derive the fundamental properties of the extension $f^{*}$ of $f$ to be defined in $\S 3$.

LEMMA 2.14. Let $f$ be an n-place operation on $A, f \in \Phi_{p}$, and let $Y$ be an ultrafilter of $\mathfrak{A}$. Assume that $V \subseteq^{n} A$ such that (a) for every $u, v \in V$ we have $u \cdot v \geqq t$ for some $t \in V$, and (b) $f v \in Y$ for every $v \in V$. Then there exists a filter $W$ of ${ }^{n} \mathfrak{U}$ such that (i) $V \cong W$, (ii) fw $\mathrm{Y}$ for every $w \in W$, and (iii) for every $j<n$ there is a set $G_{j}$ of ultrafilters of $\mathfrak{A}$ such that $\left|G_{j}\right| \leqq p$ and $\cap G_{j}=\left\{w_{j}: w \in W\right\}$.

Proof. In the algebra ${ }^{n} \mathfrak{A}$ consider the set

$$
B=\left\{x \in{ }^{n} A: f x \in Y\right\} .
$$

By hypothesis (b) we have

$$
V \sqsubseteq B
$$

Also, since $f$ is monotone and $Y$ is a filter, we see that

if $x \in B$ and $x \leqq x^{\prime}$, then $x^{\prime} \in B$. 
Using (1) and (2) with hypothesis (a), we conclude that for the filter $V^{\prime}$ generated by $V$ in ${ }^{n} \mathfrak{A}$ we have $V^{\prime} \subseteq B$. Hence, by the maximality principle of set theory, we can find a filter $W$ of ${ }^{n} \mathfrak{A}$ such that

$W$ is maximal in the class of all those filters $U$ of ${ }^{n} \mathfrak{A}$ for which $V \subseteq U \subseteq B$.

Since $V \subseteq W$ and $W \subseteq B$ by (3), we see that: Conditions (i) and (ii) of Lemma 2.14 are satisfied .

Using the maximality property of $W$ given in (3), we shall show now that (iii) is satisfied as well.

Consider any $j<n$ and set $W_{j}=\left\{w_{j}: w \in W\right\}$. Since $W$ is a filter of $n \mathfrak{A}$, obviously we have

$$
W_{j} \text { is a filter of } \mathfrak{A} \text {. }
$$

We now consider two cases.

Case 1. Suppose $0 \in W_{j}$. In this case we can take $G_{j}$ to be the empty set of ultrafilters of $\mathfrak{A}$. For each $x \in A$ we have, vacuously, $x \in X$ for every $X \in G_{j}$, so that $\cap G_{j}=A$. But by (5) and the hypothesis of Case 1 , also $W_{j}=A$. Since $\left|G_{j}\right| \leqq p$ we see that

Condition (iii) of Lemma 2.14 holds in case 1 .

Case 2. Suppose that $0 \notin W_{j}$. In this case $W_{j}$ is a proper filter of $\mathfrak{A}$, by (5), and we shall establish condition (iii) by an application of 1.3 .

Consider, therefore, any $Z \subseteq A$ such that

$$
|Z| \leqq p+1 \text { and } \Sigma Z=1 \text {, }
$$

and assume that

$$
\left(W_{j} \cap \sigma_{p} Z\right)=\varnothing \text {. }
$$

Consider any $t \in \sigma_{p} Z$, and take $x^{(t)} \in{ }^{n} A$ so that $x_{j}^{(t)}=t$ while $x_{i}^{(t)}=1$ for every $i \neq j, i<n$. By (8) we have $x^{(t)} \notin W$. Hence from (3) we conclude that the filter of ${ }^{n} A$ generated by $W \cup\left\{x^{(t)}\right\}$ is not a subset of $B$. Thus, by (2), there must be some element $w^{(t)} \in W$ for which $w^{(t)} \cdot x^{(t)} \notin B$, i.e.,

$$
w^{(t)} \in W \quad \text { and } \quad f\left(w^{(t)} \cdot x^{(t)}\right) \notin Y .
$$

Since $\sigma_{p} Z$ is finite, we can form the element $w=\Pi\left\{w^{(t)}: t \in \sigma_{p} Z\right\}$. Since $W$ and $Y$ are filters, and $f$ is monotone, we obtain from (9): 


$$
w \in W \quad \text { and } \quad f\left(w \cdot x^{(t)}\right) \notin Y \text { for every } t \in \sigma_{p} Z \text {. }
$$

Since $Z \cong \sigma_{p} Z$, we see that $x^{(z)}$ is defined for each $z \in Z$. Set $S=\left\{w \cdot x^{(z)}: z \in Z\right\}$. Then from the definition of $x^{(t)}$ we obtain

$$
\sigma_{p} S=\left\{w \cdot x^{(t)}: t \in \sigma_{p} Z\right\} .
$$

Also from the definition of $x^{(t)}$ we can see that for any $s, s^{\prime} \in S$ we have $s={ }_{j} s^{\prime}$. Since $S$ is finite and $f \in \Phi_{p}$ by hypothesis, we can therefore apply 2.4 to conclude

$$
f(\Sigma S)=\Sigma\left\{f r: r \in \sigma_{p} S\right\} .
$$

By (7) and the definition of $x^{(t)}$ we see that $\Sigma_{z \in Z} x^{(z)}=1$, and hence $\Sigma S=w$. Since $w \in B$ by (10) and (3), it follows that $f(\Sigma S) \in Y$. Since $Y$ is an ultrafilter by hypothesis, we then infer from (12) that $f r \in Y$ for some $r \in \sigma_{p} S$. Thus, by (11), we get $f\left(w \cdot x^{(t)}\right) \in Y$ for some $t \in \sigma_{p} Z$, contradicting (10).

The contradiction arises from our assumption (8). We have thus shown that for every $Z \leqq A$ such that $|Z| \leqq p+1$ and $\Sigma Z=1$, we have $\left(W_{j} \cap \sigma_{p} Z\right) \neq \varnothing$. Furthermore, by (5) we see that in Case 2, $W_{j}$ is a proper filter of $\mathfrak{A}$. Hence we can apply 1.3 to conclude that there must exist ultrafilters $X_{0}, \cdots, X_{p-1}$ such that $W_{j}=\bigcap_{i<p} X_{i}$. Putting $G_{j}=\left\{X_{0}, \cdots, X_{p-1}\right\}$ we then have:

$$
\text { Condition (iii) of Lemma } 2.14 \text { holds in Case } 2 .
$$

We see from (4), (6), and (13) that our proof is complete.

3. Throughout this section we consider an arbitrary Boolean algebra

$$
\mathfrak{U}=\langle A,+, \cdot,-, 0,1\rangle,
$$

and its Stone extension

$$
\mathfrak{A}^{*}=\left\langle A^{*}, \cup, \cap,{ }_{J} \sim, \varnothing, J\right\rangle,
$$

where $J$ is the set of all ultrafilters of $\mathfrak{A}$, and $A^{*}$ is the set of all subsets of $J$. We let $\theta$ be the canonical isomorphism whereby $\mathfrak{A}$ is represented in $\mathfrak{A}^{*}$, so that $\theta x=\{X \in J: x \in X\}$ for each $x \in A$.

Our principal task is to show how each element of $\Phi_{\omega}(A)$ may be suitably extended to an operation on $A^{*}$. Since the operations on $A^{*}$ with which we deal are $n$-place operations, we shall be concerned with various elements $G \in^{n}\left(A^{*}\right)$. With respect to such elements we shall have occasion to deal with Boolean operations upon and relations between them, referring to the Boolean algebra ${ }^{n}\left(\mathfrak{U}^{*}\right)$. Notationally, we shall write $G \subseteq{ }^{(n)} H$, for example, to indicate that $G_{j} \subseteq H_{j}$ for each 
$j<n$; or we shall use $\bigcup_{i \in I}^{(n)} G^{(i)}$ to denote the element $H$ of ${ }^{n}\left(A^{*}\right)$ such that for every $j<n$ we have $H_{j}=\bigcup_{i \in I} G_{j}^{(i)}$. Finally, if $G \in{ }^{n}\left(A^{*}\right)$, we shall frequently have occasion to refer to "subsets" $G^{\prime} \leqq{ }^{(n)} G$ which are finite, meaning thereby that for each $j<n, G_{j}^{\prime}$ is a finite subset of $G_{j}$; since $G_{j}^{\prime}$ is a finite set of ultrafilters on $A, \cap\left(G_{j}^{\prime}\right)$ is a filter, and if $x \in{ }^{n} A$, we write $x \in{ }^{(n)}\left(\bigcap^{(n)} G^{\prime}\right)$ to indicate that $x_{j} \in\left(\bigcap G_{j}^{\prime}\right)$ for each $j<n$.

Definition 3.1. To each $n$-place operation $f$ on $A$ we associate an $n$-place operation $f^{*}$ on $A^{*}$, as follows. For any $G \in{ }^{n}\left(A^{*}\right)$ we set

$$
\begin{aligned}
f^{*} G= & \left\{X \in J: \text { For some finite } G^{\prime} \subseteq^{(n)} G\right. \text { we } \\
& \text { have } \left.f y \in X \text { for every } y \in{ }^{(n)}\left(\cap^{(n)} G^{\prime}\right)\right\} .
\end{aligned}
$$

THEOREM 3.2. For every n-place operation $f$ on $A$, the operation $f^{*}$ is monotonic on $A^{*}$.

Proof. If $G, H \in{ }^{n}\left(A^{*}\right)$ and $G \leqq{ }^{(n)} H$, then whenever $G^{\prime} \cong{ }^{(n)} G$ we have also $G^{\prime} \subseteq{ }^{(n)} H$; hence $f^{*} G \subseteq f^{*} H$ by 3.2 .

Theorem 3.3. Let $f$ be any n-place operation on $A$, and $G \in{ }^{n}\left(A^{*}\right)$. If $G$ is finite, then

$$
f^{*} G=\left\{X \in J: \text { fy } \in X \text { for every } y \in \in^{(n)}\left(\bigcap^{(n)} G\right)\right\} .
$$

Proof. Whenever

$$
G^{\prime} \sqsubseteq^{(n)} G \text { and } y \in \in^{(n)}\left(\bigcap^{(n)} G\right),
$$

we have also $y \in{ }^{(n)}\left(\bigcap^{(n)} G^{\prime}\right)$. Therefore if $G$ is finite, the conclusion of 3.3 follows directly from 3.1. Then

THEOREM 3.4. Let $f$ be any n-place operation on $A$, and $G \in{ }^{n}\left(A^{*}\right)$.

$$
f^{*} G=\bigcup\left\{f^{*} G^{\prime}: G^{\prime} \leqq{ }^{(n)} G \text { and } G^{\prime} \text { is finite }\right\} .
$$

Proof. By 3.1 and 3.3.

We wish now to show that for every $f \in \Phi_{\omega}(\mathfrak{H}), f^{*}$ is an extension of $f$ with respect to the canonical embedding $\theta$ of $\mathfrak{A}$ into $\mathfrak{A}^{*}$. In this connection, if $x \in{ }^{n} A$ we use the notation $\theta^{(n)}(x)$ to indicate the element $G \in{ }^{n}\left(A^{*}\right)$ such that $\theta\left(x_{j}\right)=G_{j}$ for each $j<n$. If $G \in{ }^{n}\left(A^{*}\right)$ and $X \in{ }^{n} J$, we also use the notation $X \in^{(n)} G$ to indicate that $X_{j} \in G_{j}$ for every $j<n$.

THEOREM 3.5. Let $f$ be an n-place operation on $A, f \in \Phi_{\omega}$. Then 
for every $x \in{ }^{n} A$ we have

$$
f^{*}\left(\theta^{(n)} x\right)=\theta(f x) .
$$

Proof. Take any $x \in{ }^{n} A$. We first show that $f^{*}\left(\theta^{(n)} x\right) \leqq \theta(f x)$; for this part of the work we shall not need the hypothesis $f \in \Phi_{\omega}$.

Consider, then, any $Y \in f^{*}\left(\theta^{(n)} x\right)$. By 3.1 there exists a finite $G^{\prime} \in{ }^{n}\left(A^{*}\right)$ such that (a) $G^{\prime} \cong{ }^{(n)} \theta^{(n)} x$, and (b) $f z \in Y$ for all $z \in \in^{(n)} \cap^{(n)} G^{\prime}$. Using the definition of $\theta$, we infer from (a) that $x \in^{(n)} X$ for each $X \in{ }^{(n)} G^{\prime}$, i.e., that $x \in{ }^{(n)} \cap^{(n)} G^{\prime}$. Hence by (b) we obtain $f x \in Y$. But then $Y \in \theta(f x)$, by definition of $\theta$. This proves that $f^{*}\left(\theta^{(n)} x\right) \subseteq \theta(f x)$ as claimed.

It remains to show that $\theta(f x) \subseteq f^{*}\left(\theta^{(n)} x\right)$. For this we make use of our hypothesis that $f \in \Phi_{\omega}(\mathfrak{H})$; say $f \in \Phi_{p}$, where $p$ is a positive integer.

Now consider any $Y \in \theta(f x)$. By definition of $\theta$, this means that $f a \in Y$. Taking $V$ to be $\{x\}$, we see that we can apply Lemma 2.14 to obtain a filter $W$ of ${ }^{n} A$ such that (i) $x \in W$, (ii) $f w \in Y$ for $w \in W$, and (iii) for every $j<n$ there is some finite set $G_{j}^{\prime} \in A^{*}$ such that $\left\{w_{j}: w \in W\right\}=\bigcap G_{j}^{\prime}$.

Now let $G^{\prime}$ be the element of ${ }^{n}\left(A^{*}\right)$ determined by the sets $G_{0}^{\prime}, \cdots$, $G_{n-1}^{\prime}$. Clearly we have

(a) $G^{\prime}$ is finite.

Furthermore,

(b) $G^{\prime} \cong(n)\left(\theta^{(n)} x\right)$.

For if $X \in^{(n)} G^{\prime}$, then $x \in^{(n)} X$ by (i) and (iii) above. By definition of $\theta$, this shows that $X \in^{(n)}\left(\theta^{(n)} x\right)$, justifying (b). Finally,

( c) $f w \in Y$ for every $w \in{ }^{(n)}\left(\bigcap^{(n)} G^{\prime}\right)$

by (ii) and (iii).

Combining (a)-(c) we infer, by 3.1 , that $Y \in f^{*}\left(\theta^{(n)} x\right)$. This shows that $\theta(f x) \subseteq f^{*}\left(\theta^{(n)} x\right)$, and completes our proof.

We next wish to compute the extensions $f^{*}$ for certain special operations $f$ on $A$, each of which is in fact an element of $\Phi_{1}$.

THEOREM 3.6. Let $f$ and $g$ be the 2-place operations on $A$ such that $f x=x_{0}+x_{1}$ and $g x=x_{0} \cdot x_{1}$ for each $x \in^{2} A$. Where $n$ is any positive integer and $j<n$, let $h^{(n, j)}$ be the n-place operation of projection on $A$, such that $h^{(n, j)} x=x_{j}$ for each $x \in{ }^{n} A$. Then we have $f^{*} G=G_{0} \cup G_{1}$ and $g^{*} G=G_{0} \cap G_{1}$ for every $G \in^{2}\left(A^{*}\right)$, and we have $h^{(n, j) *} G=G_{j}$ for every $G \in{ }^{n}\left(A^{*}\right)$.

Proof. We shall give details only for the operation $f$, the proofs for the operations $g$ and $h^{(n, j)}$ being similar. 
Consider, then, any element $G$ of ${ }^{2}\left(A^{*}\right)$. By 3.1 and the definition of $f$ we have, for every $X \in J$ :

$$
X \in f^{*} G \text { if and only if there is some finite } G^{\prime} \sqsubseteq^{(2)} G
$$

$$
\text { such that }\left(y_{0}+y_{1}\right) \in X \text { for every } y \in{ }^{(2)}\left(\bigcap^{(2)} G^{\prime}\right) \text {. }
$$

Now suppose, first, that $X \in G_{0} \cup G_{1}$-without loss of generality we may suppose $X \in G_{0}$. Then take $G^{\prime}$ to be the element of ${ }^{2}\left(A^{*}\right)$ such that $G_{0}^{\prime}=\{X\}$ and $G_{1}^{\prime}=\varnothing$. Obviously $G^{\prime}$ is finite and $G^{\prime} \subseteq^{(2)} G$. Furthermore, for every $y \in{ }^{(2)}\left(\bigcap^{(2)} G^{\prime}\right)$ we have $y_{0} \in X$, and hence $\left(y_{0}+y_{1}\right) \in X$ (since $X$ is a filter). Thus we conclude by (1) that $X \in f^{*} G$. This shows that $\left(G_{0} \cup G_{1}\right) \subseteq f^{*} G$.

Conversely, consider any $X \in f^{*} G$. By (1), there exists some finite $G^{\prime} \cong{ }^{(2)} G$ such that for every $y \in{ }^{(2)}\left(\bigcap^{(2)} G^{\prime}\right)$ we have $\left(y_{0}+y_{1}\right) \in X$. We are going to show that

$$
\left(\left(\cap G_{0}^{\prime}\right) \cap\left(\bigcap G_{1}^{\prime}\right)\right) \subseteq X
$$

Indeed, since $G^{\prime} \in{ }^{2}\left(A^{*}\right)$ we see that $\cap G_{0}^{\prime}$ and $\cap G_{1}^{\prime}$ are filters of A. Given any $z \in\left(\cap G_{0}^{\prime} \cap \cap G_{1}^{\prime}\right)$, let $y$ be the element of ${ }^{2} A$ such that $y_{0}=y_{1}=z$; then $y_{0} \in \bigcap G_{0}^{\prime}, y_{1} \in \bigcap G_{1}^{\prime}$, and hence $y \in{ }^{(2)}\left(\bigcap^{(2)} G^{\prime}\right)$. It follows from our choice of $G^{\prime}$ above that $\left(y_{0}+y_{1}\right) \in X$, and hence $z \in X$. This shows that (2) holds, as claimed.

Now $G_{0}^{\prime}$ and $G_{1}^{\prime}$ are finite sets of ultrafilters of $A$. Hence we can apply Theorem 1.1 to (2) above, to conclude that $X \in G_{0}^{\prime}$ or $X \in G_{1}^{\prime}$. Since $G^{\prime} \cong{ }^{(2)} G$, this implies that $X \in\left(G_{0} \cup G_{1}\right)$. We have thus shown that $f^{*} G \leqq\left(G_{0} \cup G_{1}\right)$, completing our proof of the part of 3.6 dealing with the function $f$.

As indicated above, the proofs for $g$ and $h^{(n, j)}$ are similar, and we omit details.

THEOREM 3.7. Let $f$ be an m-place operation, $f \in \Phi_{p}(\mathfrak{A})$, and let $g_{0}, \cdots, g_{m-1}$ be $n$-place operations on A. Assume that $g_{i}$ is monotonic for each $i<m$. Put $h=f\left[g_{0}, \cdots, g_{m-1}\right]$. Then $h^{*}=f^{*}\left[g_{0}^{*}, \cdots, g_{m-1}^{*}\right]$.

Proof. Let $G$ be any element of ${ }^{n}\left(A^{*}\right)$. We shall first show that $h^{*} G \sqsubseteq f^{*}\left(g_{0}^{*} G, \cdots, g_{m-1}^{*} G\right)$.

Consider, then, any $X \in h^{*} G$. By 3.1 we can find some finite $G^{\prime} \cong{ }^{(n)} G$ such that:

$$
h y \in X \text { for every } y \in{ }^{(n)}\left(\bigcap^{(n)} G^{\prime}\right) .
$$

Now let

$$
V=\left\{v \in{ }^{m} A: v=\left\langle g_{0} y, \cdots, g_{m-1} y\right\rangle \text { for some } y \in \in^{(n)}\left(\bigcap^{(n)} G^{\prime}\right)\right\} .
$$

We have $h y=f\left(g_{0} y, \cdots, g_{m-1} y\right)$ for every $y \in{ }^{n} A$, by definition of $h$; 
hence by (1) and (2) we get

$$
f v \in X \text { for every } v \in V .
$$

We claim also that:

$$
\begin{array}{r}
\text { For every } u, v \in V \text { we have } \\
u \cdot v \geqq t \text { for some } t \in V .
\end{array}
$$

For given any $u, v \in V$, we have $u=\left\langle g_{0} y, \cdots, g_{m-1} y\right\rangle$ and $v=\left\langle g_{0} z, \cdots\right.$, $\left.g_{m-1} z\right\rangle$ for some $y, z \in{ }^{(n)}\left(\bigcap^{(n)} G^{\prime}\right)$, by (2). Since $\left(\bigcap^{(n)} G^{\prime}\right)_{j}$ is a filter of $A$ for each $j<n$, we conclude that $(y \cdot z) \in{ }^{(n)}\left(\bigcap^{(n)} G^{\prime}\right)$. Hence, setting $t=\left\langle g_{0}(y \cdot z), \cdots, g_{m-1}(y \cdot z)\right\rangle$, we have $t \in V$, by (2). Furthermore, $u \cdot v \geqq$ $t$ since each $g_{i}$ is monotonic by hypothesis. Thus (4) is justified.

Using (2) and (3), we can apply Lemma 2.14 to find a filter $W$ of ${ }^{m} A$ such that

(i) $V \subseteq W$,

(ii) $f w \in X$ for every $w \in W$, and

(iii) for every $i<m$ there is some finite set $H_{i}^{\prime} \in A^{*}$ such that $\left\{w_{i}: w \in W\right\}=\bigcap H_{i}^{\prime}$.

Now let $H^{\prime}$ be the element of ${ }^{m}\left(A^{*}\right)$ determined by the sets $H_{0}^{\prime}, \cdots, H_{m-1}^{\prime}$. Clearly we have

$$
H^{\prime} \in{ }^{m}\left(A^{*}\right) \quad \text { and } H^{\prime} \text { is finite. }
$$

Next, let $H \in{ }^{m}\left(A^{*}\right)$ be such that

$$
H_{i}=g_{i}^{*} G \text { for every } i<m \text {. }
$$

Obviously we get

$$
f^{*} H=\left(f^{*}\left[g_{0}^{*}, \cdots, g_{m-1}^{*}\right]\right) G .
$$

Now we claim that

$$
H^{\prime} \subseteq{ }^{(m)} H
$$

For consider any $Y \in^{(m)} H^{\prime}$. Then by (iii) we have $w \in^{(m)} Y$ for every $w \in W$, and hence by (i) we get $v \in^{(m)} Y$ for every $v \in V$. This means, by (2), that for any $i<m$ we have $g_{i} y \in Y_{i}$ for every $y \in{ }^{(n)}\left(\bigcap^{(n)} G^{\prime}\right)$, which shows, by 3.1 , that $Y_{i} \in g_{i}^{*} G=H_{i}$ by (6). Thus $Y \in{ }^{(m)} H$, justifying (8).

Finally, we have

$$
f w \in X \text { for every } w \in \in^{(m)}\left(\bigcap^{(m)} H^{\prime}\right),
$$

as we see by (ii) and (iii).

Combining (5), (8) and (9) we infer, by 3.1, that $X \in f^{*} H$. Thus $X \in\left(f^{*}\left[g_{0}^{*}, \cdots, g_{m-1}^{*}\right]\right) G$, by (7), concluding our demonstration that 


$$
h^{*} G \subseteq f^{*}\left(g_{0}^{*} G, \cdots, g_{m-1}^{*} G\right) .
$$

To complete our proof of 3.7 we shall now establish the opposite inclusion to that of (10).

Let $H$, as in (6), be the element of ${ }^{m}\left(A^{*}\right)$ such that $H_{i}=g_{i}^{*} G$ for every $i<m$, so that (7) holds. Consider any $X \in f^{*} H$. By 3.1 this means there is some finite $H^{\prime} \cong{ }^{(m)} H$ such that

$$
f z \in X \text { for all } z \in^{(m)}\left(\bigcap^{(m)} H^{\prime}\right) .
$$

Since $H_{i}^{\prime} \subseteq^{(m)} H_{i}=g_{i}^{*} G$ for each $\mathrm{i}<m$, we see from 3.1 that for each $Z \in{ }^{(m)} H^{\prime}$ we can find some finite $G^{(z)} \cong{ }^{(n)} G$ such that

$$
g_{i} y \in Z_{i} \quad \text { whenever } i<m \text { and } y \in \in^{(n)}\left(\bigcap^{(n)} G^{(z)}\right) .
$$

Setting $G^{\prime}=\bigcup\left\{G^{(Z)}: Z \in{ }^{(m)} H^{\prime}\right\}$, and recalling that $H^{\prime}$, as well as each $G^{(Z)}$, is finite, we see that $G^{\prime}$ is finite; and evidently we have $G^{\prime} \cong{ }^{(n)} G$. Furthermore, for any $y \in{ }^{(n)}\left(\bigcap^{(n)} G^{\prime}\right)$ we have $g_{i} y \in\left(\bigcap^{(m)} H^{\prime}\right)_{i}$ for every $i<m$, by (12), so that $f\left(g_{0} y, \cdots, g_{m} y\right) \in X$ by (11), and hence $h y \in X$ by our hypothesis on $h$. Applying 3.1, we conclude that $X \in h^{*} G$. This proves that $f^{*} H \subseteq h^{*} G$, and hence by (7) that

$$
f^{*}\left(g_{0}^{*} G, \cdots, g_{m-1}^{*} G\right) \leqq h^{*} G .
$$

Since $G$ is an arbitrary element of ${ }^{n}\left(A^{*}\right),(10)$ and (13) together complete the proof of 3.4 .

We now come to the principal theorem which relates the operations $f$ of $\Phi_{\omega}(\mathfrak{Q})$ to the corresponding operations $f^{*}$ on $A^{*}$.

Theorem 3.8. Let $f_{0}, \cdots, f_{k-1} \in \Phi_{\omega}(A)$. Suppose that

$$
\tau\left(f_{0}, \cdots, f_{k-1} ; x_{0}, \cdots, x_{n-1}\right)=\sigma\left(f_{0}, \cdots, f_{k-1} ; x_{0}, \cdots, x_{n-1}\right)
$$

is an equation which holds for all values $x_{0}, \cdots, x_{n-1} \in A$. Then the corresponding equation

$$
\tau\left(f_{0}^{*}, \cdots, f_{k-1}^{*} ; G_{0}, \cdots, G_{n-1}\right)=\sigma\left(f_{0}^{*}, \cdots, f_{k-1}^{*} ; G_{0}, \cdots, G_{n-1}\right)
$$

holds for all values $G_{0}, \cdots, G_{n-1} \in A^{*}$.

Proof. Corresponding to each of the two sides $\tau$ and $\sigma$ of the original equation there are $n$-place operations $h$ and $g$ on $A$, each obtained from $f_{0}, \cdots, f_{k-1}$ (and from the projections $h^{(n, j)}$ of 3.6 ) by an indicated series of compositions. The hypothesis that the equation $\tau=\sigma$ holds identically in $A$, implies that in fact $h=g$.

In the algebra $A^{*}$, the two sides $\tau$ and $\sigma$ of the equation determine $n$-place operations, obtained from $f_{0}^{*}, \cdots, f_{k-1}^{*}$ by performing the same 
indicated series of compositions as were used in obtaining $h$ and $g$; the resulting operations are, in fact, $h^{*}$ and $g^{*}$, as we see by repeated application of 3.7 (and by 3.6). Since we have $h=g$, of course we infer that $h^{*}=g^{*}$. But this implies that the equation $\tau=\sigma$ holds identically in $A^{*}$, as claimed in the theorem.

\section{Theorem 3.9. If $f \in \Phi_{p}(\mathfrak{I})$, then $f^{*} \in \Phi_{p}^{c}\left(\mathfrak{U}^{*}\right)$.}

Proof. Let $f$ be any $n$-place operation on $A, f \in \Phi_{p}(\mathfrak{U})$. As remarked in 2.2 , the set of those $n$-place operations which are in $\Phi_{p}$ can be characterized by means of $n$ certain equational identities. Hence by 3.8 we see that

$$
f^{*} \in \Phi_{p}\left(\mathfrak{I}^{*}\right) .
$$

Now consider any $G \in{ }^{n}\left(A^{*}\right)$, and let

$$
A^{*^{(G)}}=\left\{G^{\prime} \in^{n}\left(A^{*}\right): G^{\prime} \leqq{ }^{(n)} G \text { and }\left|G_{j}\right| \leqq 1 \text { for every } j<n\right\} \text {. }
$$

Take any $X \in f^{*} G$. By 3.4 we can find some $G^{\prime} \in{ }^{n}\left(A^{*}\right)$ such that

$$
X \in f^{*} G^{\prime} \quad \text { and } G^{\prime} \text { is finite. }
$$

From the latter we get a positive integer $q$ such that

$$
G^{\prime}=\bigcup_{i<q}^{(n)} G^{(i)} \text { for some } G^{(0)}, \cdots, G^{(q-1)} \in A^{*(G)} .
$$

Now using (1), let us apply 2.4 to the operation $f^{*}$ on the algebra $A^{*}$; then by (4) we get

$$
f^{*} G^{\prime}=\bigcup\left\{f^{*} H: H \in \sigma_{p}\left\{G^{(0)}, \cdots, G^{(q-1)}\right\}\right\},
$$

whence by (3) and (4),

$$
X \in f^{*} H \text { for some } H \in \sigma_{p}\left(A^{*(G)}\right) .
$$

Since $X$ is an arbitrary element of $f^{*} G$, this shows that

$$
f^{*} G \subseteq \bigcup\left\{f^{*} H: H \in \sigma_{p}\left(A^{*(G)}\right)\right\} .
$$

The opposite inclusion also holds by 3.2 , since for each $H \in \sigma_{p}\left(A^{*(G)}\right)$ we have $H \leqq{ }^{(n)} G$ by (2). Thus

$$
f^{*} G=\bigcup\left\{f^{*} H: H \in \sigma_{p}\left(A^{*\left(G^{*}\right.}\right)\right\} \text {. }
$$

By applying 2.11 we infer from (7) and (2) that $f^{*} \in \Phi_{p}^{c}\left(\mathfrak{2}^{*}\right)$, as desired.

REMARK 3.10. We can summarize the combined substance of 3.5 , 3.8 and 3.9 by saying that each $p$-additive operation $f$ on $A$ can be extended to a completely $p$-additive operation $f^{*}$ on $A^{*}$, in such a 
way that whenever an equation in given operations $f_{0}, \cdots, f_{m-1}$ holds identically in $\mathfrak{A}$, the corresponding equation in the operations $f_{0}^{*}, \cdots$, $f_{m-1}^{*}$ holds identically in $\mathfrak{U}^{*}$. If we restrict this result to the case $p=1$, we get a result of Jonsson-Tarski [2]. (Cf. remark preceding Theorem 2.11 of that paper.)

Following Jonsson and Tarski further, we may generalize their notion of a Boolean algebra with operators. Let us call a system

$$
\mathfrak{B}=\left\langle B,+, \cdot,-, 0,1, f_{0}, \cdots, f_{m-1}\right\rangle
$$

a Boolean algebra with partial operators if $\langle B,+, \cdot,-, 0,1\rangle$ is a Boolean algebra and $f_{0}, \cdots, f_{m-1} \in \Phi_{\omega}(\mathfrak{B})$. Such a system $B$ will be called atomistic if its Boolean part is atomistic; $B$ will be called complete if its Boolean part is complete and $f_{0}, \cdots, f_{m-1} \in \Phi_{\omega}^{c}$.

Suppose that we define a class $\mathscr{L}$ of structures by means of a system of axioms each having the form of an equational identity. Then by $3.5,3.8$ and 3.9 we can conclude:

If every structure of $\mathscr{L}$ is a Boolean algebra with partial operators, then each $\mathfrak{B} \in \mathscr{L}$ can be extended to a complete, atomistic $\mathfrak{B}^{*} \in \mathscr{L}$.

If we start with a Boolean algebra $\mathfrak{A}$ which is complete, the extension Theorem 3.5 provides us with a simple method of constructing all operations of $\Phi_{\omega}(\mathfrak{H})$. The method of construction is described in the following definition.

Definition 3.11. For any positive integers $n$ and $p$, let

$$
\begin{aligned}
A_{p}^{*} & =\left\{G \in A^{*}:|G| \leqq p\right\}, \\
\Gamma_{n, p} & =\text { the set of all functions mapping } A_{p}^{*} \text { into } A^{*} .
\end{aligned}
$$

Now assume that $\mathfrak{U}$ is complete. Then for each $\alpha \in \Gamma_{n, p}$ define $f_{\alpha}$ to be the $n$-place operation on $A$ such that, for any $x \in{ }^{n} A$ we have:

$$
\left(^{*}\right) \quad f_{\alpha} x=\Pi \cap \bigcup\left\{\alpha G: G \in{ }^{n}\left(A_{p}^{*}\right) \text { and } x \in{ }^{(n)}\left(\bigcap^{(n)} G\right)\right\} \text {. }
$$

In case $\mathfrak{A}$ is not complete, we define $f_{\alpha}$ only for those $\alpha \in \Gamma_{n, p}$ such that, for every $x \in{ }^{n} A$, the product indicated on the right side of equation $\left({ }^{*}\right)$ exists in $\mathfrak{2}$.

THEOREM 3.12. Let $n, p$ be positive integers and $\alpha \in \Gamma_{n, p}$. If $f_{\alpha}$ is defined on $\mathfrak{A}$ (in particular, if $\mathfrak{A}$ is complete), then $f_{\alpha} \in \Phi_{p}(\mathfrak{U})$.

Proof. Consider any finite $X \subseteq{ }^{n} A$, and let $X^{+}=X_{0} \times \cdots \times X_{n-1}$. For any $G \in{ }^{n}\left(A_{p}^{*}\right)$ we obtain, by 1.4 : 


$$
\begin{aligned}
(\Sigma X) \in^{(n)}\left(\bigcap^{(n)} G\right) & \text { if and only if } \\
y \in \in^{(n)}\left(\bigcap^{(n)} G\right) & \text { for some } y \in \sigma_{p}\left(X^{+}\right) .
\end{aligned}
$$

Hence, if we set

$$
H=\bigcup\left\{\alpha G: G \in{ }^{n}\left(A_{p}^{*}\right) \text { and } \Sigma X \in{ }^{(n)}\left(\bigcap^{(n)} X\right)\right\}
$$

and, for each $y \in \sigma_{p}\left(X^{+}\right)$,

$$
K^{(y)}=\bigcup\left\{\alpha G: G \in{ }^{n}\left(A_{p}^{*}\right) \text { and } y \in{ }^{(n)}\left(\bigcap^{(n)} G\right)\right\},
$$

we obtain from (1) that

$$
H=\bigcup_{y \in \sigma_{p}\left(X^{+}\right)} K^{(y)} .
$$

Since $H$ and each $K^{(y)}$ are sets of ultrafilters, we derive from (4) that

$$
\bigcap H=\bigcap_{y \in \sigma_{p}\left(X^{+}\right)}\left(\bigcap K^{(y)}\right),
$$

then, since $\sigma_{p}\left(X^{*}\right)$ is finite and $\cap H$, as well as each $\cap K^{(y)}$, is a filter, we get

$$
\Pi \cap H=\Sigma_{y \in \sigma_{p}\left(X^{+}\right)} \Pi \cap K^{(y)} .
$$

Combining (6) with (2) and (3), and comparing the result with 3.11 $(*)$, we find that

$$
f_{\alpha}(\Sigma X)=\Sigma_{y \in \sigma_{p}\left(X^{+}\right)}\left(f_{\alpha} y\right),
$$

which shows that $f_{\alpha} \in \Phi_{p}(\mathfrak{H})$ by 2.8 .

THEOREM 3.13. For every n-place $f \in \Phi_{p}(\mathfrak{X})$ there exists some $\alpha \in \Gamma_{n, p}$ such that $f_{\alpha}$ is defined on $A$ and $f_{\alpha}=f$.

Proof. Let $f$ be an $n$-place operation, $f \in \Phi_{p}$, and take $\alpha \in \Gamma_{n, p}$ so that $\alpha G=f^{*} G$ for every $G \in A_{p}^{*}$. For any $x \in{ }^{n} A$, set

$$
H^{(x)}=\bigcup\left\{f^{*} G: G \in{ }^{n}\left(A_{p}^{*}\right) \text { and } x \in \in^{(n)}\left(\bigcap^{(n)} G\right)\right\} \text {. }
$$

Clearly $H^{(x)} \in A^{*}$, and hence $\bigcap H^{(x)}$ is a filter of $\mathfrak{A}$. In order to show, for our chosen $\alpha$, that $f_{\alpha}$ is defined on $\mathfrak{A}$ and that $f_{\alpha}=f$, we see from 3.11 that for every $x \in{ }^{n} A$ we must show:

$$
f x=\Pi \cap H^{(x)} \text {. }
$$

Consider, therefore, any $x \in{ }^{n} A$. Since every $G \in A_{p}^{*}$ is finite, by 3.11, we then see from (1) and 3.3 that for every $X \in J$ we have $X \in H^{(x)}$ if and only if there exists some $G \in{ }^{n}\left(A_{p}^{*}\right)$ such that $x \in{ }^{(n)}\left(\bigcap^{(n)} G\right)$ and for every $y \in \epsilon^{(n)}\left(\cap^{(n)} G\right)$ we have $f y \in X$. 
From this we obviously have

$$
f x \in \bigcap H^{(x)} .
$$

Now consider any $z \in A$ such that

$$
(f x) \cdot-z \neq 0,
$$

and find an ultrafilter $Y$ of $\mathfrak{A}$ such that

$$
((f x) \cdot-z) \in Y \text {. }
$$

Putting $V=\{x\}$ we see, from (5), that $f v \in Y$ for each $v \in V$. Since $f \in \Phi_{p}$, by hypothesis, we can apply 2.14 to obtain a filter $W$ of ${ }^{n} \mathfrak{A}$ such that (i) $x \in W$, (ii) $f w \in W$ for every $w \in W$, and (iii) there is some $G \in{ }^{n}\left(A_{p}^{*}\right)$ such that $\left(\bigcap^{(n)} G\right)_{j}=\left\{w_{j}: w \in W\right\}$ for each $j<n$. We can, therefore, conclude from (2) that

$$
Y \in H^{(x)} \text {. }
$$

Since $Y$ is an ultrafilter, we see from (5) that $z \notin Y$ and hence, by (6), $z \notin \bigcap H^{(x)}$. But by (4), $z$ is any element of $A$ such that $(f x) \cdot-z \neq 0$. Thus we have shown that

$$
\text { For every } z \in \bigcap H^{(x)} \text { we have } f x \leqq z .
$$

Combing (7) with (3) we obtain $f x=\Pi \cap H^{(x)}$, the equation $\left({ }^{*}\right)$, which completes our proof.

REMARK 3.14. Theorems 3.12 and 3.13 together show that if $\mathfrak{A}$ is a complete Boolean algebra, then the set $\Phi_{p}(\mathfrak{H})$ coincides with the set of all operations $f_{\alpha}$ obtained from functions $\alpha \in \bigcup_{n} \Gamma_{n, p}$, the union being taken over all positive integers $n$.

Of course it may well happen that we have $\alpha, \beta \in \Gamma_{n, p}$ and $\alpha \neq \beta$, even though $f_{\alpha}=f_{\beta}$. It is not hard to see, from 3.11, that we will have $f_{\alpha}=f_{\beta}$ if and only if for every $G \in{ }^{n}\left(A_{p}^{*}\right)$ we have:

$$
\Sigma\left\{\alpha H: H \subseteq{ }^{(n)} G\right\}=\Sigma\left\{\beta H: H \subseteq{ }^{(n)} G\right\} .
$$

From this it easily follows that for every $\alpha \in \Gamma_{n, p}$ there is one and only one $\beta \in \Gamma_{n, p}$ such that $f_{\alpha}=f_{\beta}$ and $\beta$ is monotonic (i.e., $\beta H \leqq \beta G$ whenever $G \in \in^{n}\left(A_{p}^{*}\right)$ and $\left.H \subseteq{ }^{(n)} G\right)$. Thus the set of $n$-place operations in $\Phi_{p}(\mathfrak{U})$ is in one-to-one correspondence with the set of monotonic functions in $\Gamma_{n, p}$.

The representation of operations in $\Phi_{p}(\mathfrak{H})$, which is provided by 3.13, allows us to obtain an analogue to 2.12 for these operationsbut only in the case where $\mathfrak{A}$ is complete. 
THEOREM 3.15. Let $\mathfrak{A}$ be a complete Boolean algebra, let $n, p$ be positive integers, and set $q=n \cdot p$. There is a sequence $h^{\left(n, s_{0}\right)}, \cdots$, $h^{\left(n, s_{q-1}\right)}$ of projections of ${ }^{n} A$ onto $A$, such that every n-place operation $f \in \Phi_{p}(\mathfrak{U})$ can be obtained from some q-place operation $g \in \Phi_{1}(\mathfrak{U})$ by the composition

$$
f=g\left[h^{\left(n, s_{0}\right)}, \cdots, h^{\left(n, s_{q-1}\right)}\right] .
$$

Proof. As in 2.12 , take $s \in{ }^{q}\{0, \cdots, n-1\}$ so that

$$
s_{j \cdot p+i}=j \text { for every } j<n \text { and } i<p .
$$

Now consider any $f \in \Phi_{p}(\mathfrak{H})$ and take $\alpha \in \Gamma_{q, 1}$ so that, for every $G \in{ }^{q}\left(A_{1}^{*}\right)$,

$$
\alpha G=f^{*}\left(\bigcup_{i<p} G_{i}, \bigcup_{i<p} G_{p+i}, \cdots, \bigcup_{i<p} G_{(n-1) \cdot p+i}\right) .
$$

Then by (1), (2), and 3.11, we see that for every $y \in{ }^{q} A$ we have

$$
\begin{gathered}
g_{\alpha} y=\Pi \cap \bigcup\left\{f^{*}\left(\bigcup_{i<p} G_{i}, \cdots, \bigcup_{i<p} G_{(n-1) \cdot p+i}\right):\right. \\
\left.G \in{ }^{q}\left(A_{1}^{*}\right) \text { and } y \in{ }^{(q)}\left(\bigcap^{(q)} G\right)\right\} .
\end{gathered}
$$

Now for any $x \in{ }^{n} A$ let $y^{(x)}$ be the element of ${ }^{q} A$ such that

$$
y_{k}^{(x)}=h^{\left(n, s_{k}\right)} x \text { for } k=0, \cdots, q-1 \text {. }
$$

Thus

$$
\left(g_{\alpha}\left[h^{\left(n, s_{0}\right)}, \cdots, h^{\left(n, s_{q-1}\right)}\right]\right) x=g_{\alpha} y^{(x)} \quad \text { for } \quad x \in{ }^{n} A .
$$

Now consider any $x \in{ }^{n} A$ and any $G \in{ }^{q}\left(A_{1}^{*}\right)$ such that $y^{(x)} \in{ }^{(q)}\left(\bigcap^{(q)} G\right)$, and let $H \in{ }^{n}\left(A^{*}\right)$ be such that $H_{j}=\mathbf{U}_{i<p} G_{j \cdot p+i}$ for each $j<n$. Then clearly $H \in{ }^{n}\left(A_{p}^{*}\right)$ by 3.11 , and $x \in{ }^{(n)}\left(\bigcap^{(n)} H\right)$ since, by (1) and (4), $y_{j \cdot p+i}^{(x)}=x_{j}$ for any $j<n$ and $i<p$. This, together with (3), shows that, for any $x \in{ }^{n} A$,

$$
g_{\alpha} y^{(x)} \leqq \Pi \cap \bigcup\left\{f^{*} H: H \in{ }^{n}\left(A_{p}^{*}\right) \text { and } x \in{ }^{(n)}\left(\bigcap^{(n)} H\right)\right\} \text {. }
$$

On the other hand, given $x \in{ }^{n} A$ and any $H \in{ }^{n}\left(A_{p}^{*}\right)$ such that $x \in{ }^{(n)}\left(\bigcap^{(n)} H\right)$, we can, by 3.11 , find ultrafilters $X_{i}^{(j)}$ for each $j<n$ and $i<p$ such that $H_{j}=\left\{X_{0}^{(j)}, \cdots, X_{p-1}^{(j)}\right\}$, and we can then form $G \in{ }^{q}\left(A^{*}\right)$ so that $G_{j \cdot p+i}=\left\{X_{i}^{(j)}\right\}$ for each $j<n, i<p$. Clearly we will have $G \in{ }^{q}\left(A_{1}^{*}\right)$ by 3.11 , and $y^{(x)} \in{ }^{(q)}\left(\bigcap^{(q)} G\right)$ since $y_{j \cdot p+i}^{(x)}=x_{j}$ by (4) and (1). This shows that

$$
\Pi \cap \bigcup\left\{f^{*} H: H \in{ }^{n}\left(A_{p}^{*}\right) \text { and } x \in{ }^{(n)}\left(\bigcap^{(n)} H\right)\right\} \leqq g_{\alpha} y^{(x)}
$$

by (3), since clearly $H_{j}=\bigcup_{i<p} G_{j \cdot p+i}$ for each $j<n$.

Now by $3.9,3.11$, and 2.1 we have 


$$
\begin{aligned}
\bigcup & \left\{f^{*} H: H \in{ }^{n}\left(A_{p}^{*}\right) \text { and } x \in{ }^{(n)}\left(\bigcap^{(n)} H\right)\right\} \\
& =f^{*}\left(\mathbf{U}^{(n)}\left\{K \in{ }^{n}\left(A_{1}^{*}\right): x \in{ }^{(n)}\left(\bigcap^{(n)} K\right)\right\}\right. \\
& =f^{*}\left(\theta^{(n)} x\right) \quad \text { by definition of } \theta^{(n)} .
\end{aligned}
$$

Hence by (6) and (7) we get

$$
\begin{aligned}
g_{\alpha} y^{(x)} & =I \cap f^{*}\left(\theta^{(n)} x\right) \\
& =I \cap \theta(f x) \quad \text { by } 3.5 .
\end{aligned}
$$

But by definition of $\theta, \cap \theta(f x)$ is the principal filter of $A$ which is generated by $f x$, hence (8) gives

$$
g_{\alpha} y^{(x)}=f x .
$$

Since (9) holds for arbitrary $x \in{ }^{n} A$, we can combine it with (5) to obtain the equation $\left(^{*}\right)$ of 3.15 .

Problem 3.16. The question whether 3.15 can be strengthened to cover arbitrary Boolean algebras, instead of only complete ones, is open.

REMARK 3.17. In [2], Jonsson and Tarski achieve their extension theorem (cf. 3.10) by associating with each $n$-place operation $f$ on $\mathfrak{A}$ an $n$-place operation $f^{+}$on $\mathfrak{Y}^{*}$ which in general is different from $f^{*}$. Let us call an element $G \in A^{*}$ closed if and only if for every $X \in J$ such that $\bigcap G \subseteq X$, we have $X \in G$; and call $G \in{ }^{n}\left(A^{*}\right)$ closed if and only if $G_{j}$ is closed for each $j<n$. Then for each $n$-place operation $f$, the definition of $f^{+}$is given by

$$
\begin{aligned}
f^{+} G= & \left\{X \in J: \text { For some closed } G^{\prime} \leqq(n) G\right. \text { we } \\
& \text { have } \left.f y \in X \text { for every } y \in{ }^{(n)}\left(\bigcap^{(n)} G^{\prime}\right)\right\}
\end{aligned}
$$

for every $G \in^{n}\left(A^{*}\right)$.

Clearly from (1) we obtain, for each $G \in{ }^{n}\left(A^{*}\right)$,

$$
\begin{aligned}
f^{*} G= & \left\{X \in J: \text { For some filter } Y \supseteqq{ }^{(n)}\left(\bigcap^{(n)} G\right)\right. \\
& \text { we have } \left.f y \in X \text { for every } y \in{ }^{(n)} Y\right\} .
\end{aligned}
$$

If we consider, therefore, the 1-place operation $f$ on $A$ such that $f 1=$ 1 and $f x=0$ for all $x \in A$ such that $x \neq 1$, we get $f^{+} J=J$ (by taking $Y=\{1\}$ in (2)). But if $A$ is infinite, we get $f^{*} J=\varnothing$ by 3.1.

While this example shows that we can have $f^{+} \neq f^{*}$, whenever

${ }^{3}$ Actually [2] deals not with the particular Stone extension which we have called $\mathfrak{2} *$, but with an arbitrary extension $\mathfrak{B}$, of a kind called "perfect extension" by Jonsson and Tarski. In fact, as they point out, every perfect extension of $\mathfrak{A}$ is isomorphic to $\mathfrak{A} *$. In 3.17 we have adapted the description of $f^{+}$as given in [2], so as to make it applicable to the particular extension $\mathfrak{U} *$. 
$f \in \Phi_{\omega}$ we get $f^{+}=f^{*}$. This can be obtained by applying 2.14 to (2) to get $f^{+} G \subseteq f^{*} G$. The opposite inclusion holds for arbitrary $f$, since each finite $G \in{ }^{n}\left(A^{*}\right)$ is closed, by 1.1 .

4. There are two ways in which we may try to strengthen the results of the preceding section. First, we may seek a set of operations, containing $\Phi_{\omega}$ as a proper subset, to which we can extend such Theorems as 3.5, 3.8, and-in some fashion-3.9. Second, we may try to enlarge the scope of 3.8 to include other sentences, besides the equational identities described there, which are preserved in passing from $\mathfrak{A}$ to $\mathfrak{2}^{*}$. Actually, both of these ways are possible.

Let us consider, first, the problem of enlarging $\Phi_{\omega}$. An examination of the proofs of 3.5,3.8, and 3.9 shows that they all rest on Lemma 2.14. This lemma, in turn, is based on the following property possessed by each $n$-place operation $f \in \Phi_{p}$ : There exists a positive integer, $p$, such that whenever $Y$ is an ultrafilter of $\mathfrak{A}, X$ is a finite subset of ${ }^{n} A$, and $f(\Sigma X) \in Y$, then we have $f t \in Y$ for some $t \in \sigma_{p} X$.

The fact that the integer $p$ is uniform for all ultrafilters $Y$ is not essential for deriving the lemma, except for that part of the conclusion which specifies that the sets $G_{j}$ satisfy the condition $\left|G_{j}\right| \leqq p$. If we are willing to weaken this part of the conclusion to assert simply that each $G_{j}$ is finite, we can weaken the hypothesis by letting $p$ depend on $Y$. This observation motivates the following definition in which $\mathfrak{A}$, as in earlier sections, is assumed to be an arbitrary Boolean algebra.

Definition 4.1. We define $\psi(\mathfrak{U})$ to be the set of all those operations $f$ on $A$, of any number $n$ of places, such that for every ultrafilter $Y$ of $\mathfrak{A}$ there exists a positive integer $p$ with the following property: Whenever $X$ is a finite subset of ${ }^{n} A$ such that $f(\Sigma X) \in Y$, then $f t \in Y$ for some $t \in \sigma_{p} X$.

Obviously $\Phi_{\omega}(\mathfrak{U}) \subseteq \psi(\mathfrak{U})$. That this inclusion is proper can be seen from the following example.

ExAmple 4.2. Let $P$ be the set of all positive integers and $\mathfrak{B}$ the Boolean set algebra of all subsets of $P$. Let $f$ be the 1-place operation on $B$ such that, for any $x \subseteq P$,

$$
f x=\left\{2^{i}: i \in x\right\} \cup\left\{3^{j}: j \leqq|x|\right\},
$$

it being understood that if $x$ is infinite then all positive powers of 3 are in $f x$.

Now $f \notin \Phi_{\omega}$. For given any positive integer $q$, let $X=\{\{1\}, \cdots$, 
$\{q+1\}\}$. Obviously $3^{q+1} \in f(\mathbf{U} X)$, but $3^{q+1} \in \mathbf{U}\left\{f t: t \in \sigma_{q} X\right\}$; hence $f \notin \Phi_{q}$.

However, $f \in \Psi$. For consider any ultrafilter $Y$ of $\mathfrak{B}$. Two cases must be distinguished.

Case 1. Suppose $Y$ is a principal filter, generated by an atom $\{k\}$ of $\mathfrak{B}$. (i) In case $k$ is neither a power of 2 nor a power of 3 , then $f x \notin Y$ for all $x \in B$; in this case we put $p=1$. (ii) In case $k=2^{i}$, then we have $f x \in Y$ if and only if $i \in x$; in this case we again put $p=1$; (iii) In case $k=3^{j}$, then we have $f x \in Y$ if and only if $|x| \geqq j$; in this case we put $p=j$. It is now readily seen that under any of (i), (ii), or (iii), whenever we have $X \subseteq B$ and $f(\bigcup X) \in Y$, then we must have also $f t \in Y$ for some $t \in \sigma_{p} X$.

Case 2. Suppose $Y$ is a nonprincipal ultrafilter. In this case we have $f x \in Y$ whenever $x$ is finite. Thus we can be assured that if $X$ is a finite subset of $B$ such that $f(\bigcup X X) \in Y$, then $f x \in Y$ for some $x \in X$. We can, therefore, take $p=1$, and the condition for $f$ to be in $\Psi$ will be satisfied.

Having seen that $\Psi$ is a proper extension of $\Phi_{\omega}$, it is now a simple matter to prove a modified form of Lemma 2.14 as indicated immediately preceding 4.1. Based upon this, we can then obtain the following theorem generalizing $3.5,3.8$, and 3.9 .

Theorem 4.3. (i) For every n-place $f \in \Psi$ we have

$$
f^{*}\left(\theta^{(n)} x\right)=\theta(f x) \text {. }
$$

(ii) If $f_{0}, \cdots, f_{k-1} \in \Psi$, and if an equation

$$
\tau\left(f_{0}, \cdots, f_{k-1} ; x_{0}, \cdots, x_{m-1}\right)=\sigma\left(f_{0}, \cdots, f_{k-1} ; x_{0}, \cdots, x_{m-1}\right)
$$

holds for all values $x_{0}, \cdots, x_{m-1} \in A$, then the equation

$$
\tau\left(f_{0}^{*}, \cdots, f_{i-1}^{*} ; G_{0}, \cdots, G_{m-1}\right)=\sigma\left(f_{0}^{*}, \cdots, f_{k-1}^{*} ; G_{0}, \cdots, G_{m-1}\right)
$$

holds for all values $G_{0}, \cdots, G_{m-1} \in A^{*}$.

(iii) For each atom $H$ of $\mathfrak{I}^{*}$, there is a positive integer $p$ with the following property: Whenever $\Gamma \leqq A^{*}$ and $H \in f^{*}(\bigcup \Gamma)$, then we have $H \in f^{*} G$ for some $G \in \sigma_{p} \Gamma$.

Proof. Using the modified form of Lemma 2.14 instead of the original, it is a simple matter to alter the proofs of 3.5,3.8, and 3.9, so as to derive 4.3. We omit details.

We turn now to the question of improving 3.8 , or its generalization 
4.3 (ii), by enlarging the set of equations $\tau=\sigma$ which are shown to be preserved in passage from $A$ to $A^{*}$. Among the operations which may be used in forming the two sides $\tau$ and $\sigma$ of such an equation are, of course, the Boolean operations + and $\cdot$, since these are obviously in $\Phi_{\omega}$-and as shown in 3.6, their extensions to $\mathfrak{I}^{*}$ are the Boolean operations $U$ and $\cap$ of this algebra. However, the Boolean operation -is not in $\Phi_{\omega}$, nor in $\psi$, and so may not appear in the equations covered by 3.8 or 4.3 (ii). As Jonsson and Tarski have shown, there are equations involving the Boolean operation - and an additive operation $f$, which may hold identically in some Boolean algebra but not in its Stone extension-or in any complete Boolean algebra. However, we shall indicate below a class of equations in which - may appear together with operations of $\Psi$, to which 4.3 (ii) can be extended.

In order to obtain this result we first take up and elaborate the observation of Jonsson and Tarski that their results can be extended to cover functions from one Boolean algebra to another. In fact, still more generally, we can consider $n$-place mappings into a Boolean algebra, $\mathfrak{B}$, in which each of the $n$ arguments ranges over a distinct Boolean algebra, $\mathfrak{Y}_{\jmath}$. The generalization of the basic definitions and theorems of $\S 2$ and $\S 3$ to cover such mappings presents no difficulty. In place of the sets $\Phi_{\omega}(A)$ we will have sets $\Phi_{\omega}\left(\mathfrak{A}_{0}, \cdots, \mathfrak{A}_{n-1} ; \mathfrak{B}\right)$, and similarly for $\Psi$. When dealing with the composition of mappings in order to generalize 3.7 , we consider an $n$-place function $f$ mapping $B_{0} \times \cdots \times$ $B_{n-1}$ into $C$, and $n m$-place functions $g_{0}, \cdots, g_{n-1}$ such that $g_{i}$ maps $A_{0} \times \cdots \times A_{m-1}$ into $B_{i}$ for each $i<n-1$, and we form the composed $m$-place function $f\left[g_{0}, \cdots, g_{n-1}\right]$ mapping $A_{0} \times \cdots \times A_{m-1}$ into $C$. Similarly, in the equations to be dealt with when generalizing 3.8 or 4.3 (ii), each variable may range over the elements of a different Boolean algebra, the two sides of the equation will take on values in one Boolean algebra (which may, however, differ from the ranges of the several variables), and the various operations occurring in the equation may involve intermediate algebras.

These multi-algebra mappings are relevant to equations over a single algebra, $\mathfrak{A}=\langle A,+, \cdot,-, 0,1\rangle$, because although the operation - is not in $\Phi_{\omega}(\mathfrak{U})$ it is an additive mapping of $\mathfrak{U}$ into the dual algebra $\mathfrak{A}_{d}=\langle A, \cdot,+,-, 1,0\rangle$, and hence $-\in \Phi_{1}\left(\mathfrak{I} ; \mathfrak{H}_{d}\right)$. We can thus construct equations in which all variables and terms take their values in the set $A$, with operations including -, from any or all of the sets $\Psi(\mathfrak{U}), \Psi\left(\mathfrak{U}_{d}\right), \Psi\left(\mathfrak{U} ; \mathfrak{U}_{d}\right)$, and $\Psi\left(\mathfrak{U}_{d} ; \mathfrak{U}\right)$, and still other sets involving functions of more than one argument, such as $\Psi\left(\mathfrak{U}, \mathfrak{I}_{d} ; \mathfrak{U}_{d}\right)$. If these operations are composed properly, we can obtain equations falling within the scope of Theorem 4.3 (ii) as generalized to the multialgebra case.

Of course in applying these results to dual algebras it should be 
kept in mind that an ultrafilter of $\mathfrak{A}_{d}$ is a maximal proper ideal of $\mathfrak{A}$, so that while the algebras $\mathfrak{A}$ and $\mathfrak{U}_{d}$ have the same set of elements, $\mathfrak{A}^{*}$ and $\mathfrak{Y}_{d}^{*}$ do not. The mapping -* of $\mathfrak{U}^{*}$ into $\mathfrak{A}_{d}^{*}$ is such that for any $G \in A^{*}$ and any $I \subseteq A$ we have

$$
\begin{aligned}
& I \in\left(-{ }^{*} G\right) \text { if and only if } \\
& \quad I=\{x \in A:-x \in X\} \text { for some } X \in G .
\end{aligned}
$$

Theorem 3.5, for the operation -, takes the form:

$$
\text { - }^{*}(\theta x)=\theta_{d}(-x) \text { for all } x \in A,
$$

where, for any $y \in A, \theta_{d} y$ is the set of all these maximal proper ideals of $\mathfrak{U}$ for which $y \notin I$.

\section{BIBLIOGRAPHY}

1. L. Henkin, Extending Boolean operations, Abstracts of brief scientific commun1cations, Section 1, Mathematical logic and foundations of mathematics, International Congress of Mathematicians, Moscow, 1966.

2. B. Jonsson and A. Tarski, Boolean algebras with operators, Part I, Amer. J. Math. 73 (1951), 891-939. Part II, Amer. J. Math. 74 (1952), 127-162.

Received August 2, 1969. Some of the ideas of this paper were presented at the International Congress of Mathematicians held in Moscow in 1966. See [1]. This paper was written while the author was on sabbatical leave at Oxford University; the use of facilities of All Souls College and of the Mathematical Institute there are gratefully acknowledged. A part of the support for this work was furnished by the National Science Foundation, Grant No. GP 6232X2.

UNIVERSity of CALIFORNIA, BerkELEY, AND

OXFORD UNIVERSITY 


\title{
PACIFIC JOURNAL OF MATHEMATICS
}

\author{
EDITORS
}

H. SAMELSON

Stanford University

Stanford, California 94305

J. DugunduI

Department of Mathematics

University of Southern California

Los Angeles, California 90007

RICHARD PIERCE

University of Washington

BASIL GORDON*

University of California

Seattle, Washington 98105

Los Angeles, California 90024

\section{ASSOCIATE EDITORS}

E. F. BeCKenbaCh

B. H. NeumanN

F. WOLF

K. YosHIDA

\section{SUPPORTING INSTITUTIONS}

\author{
UNIVERSITY OF BRITISH COLUMBIA \\ CALIFORNIA INSTITUTE OF TECHNOLOGY \\ UNIVERSITY OF CALIFORNIA \\ MONTANA STATE UNIVERSITY \\ UNIVERSITY OF NEVADA \\ NEW MEXICO STATE UNIVERSITY \\ OREGON STATE UNIVERSITY \\ UNIVERSITY OF OREGON \\ OSAKA UNIVERSITY \\ UNIVERSITY OF SOUTHERN CALIFORNIA
}

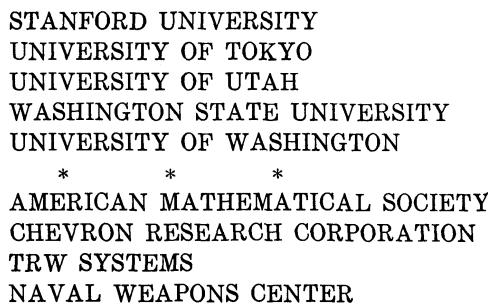

The Supporting Institutions listed above contribute to the cost of publication of this Journal, but they are not owners or publishers and have no responsibility for its content or policies.

Mathematical papers intended for publication in the Pacific Journal of Mathematics should be in typed form or offset-reproduced, double spaced with large margins. Underline Greek letters in red, German in green, and script in blue. The first paragraph or two must be capable of being used separately as a synopsis of the entire paper. It should not contain references to the bibliography. Manuscripts, in duplicate if possible, may be sent to any one of the four editors. Please classify according to the scheme of Math. Rev. 36, 1539-1546. All other communications to the editors should be addressed to the managing editor, Richard Arens, University of California, Los Angeles, California, 90024.

50 reprints are provided free for each article; additional copies may be obtained at cost in multiples of 50 .

The Pacific Journal of Mathematics is published monthly. Effective with Volume 16 the price per volume (3 numbers) is $\$ 8.00$; single issues, $\$ 3.00$. Special price for current issues to individual faculty members of supporting institutions and to individual members of the American Mathematical Society: $\$ 4.00$ per volume; single issues $\$ 1.50$. Back numbers are available.

Subscriptions, orders for back numbers, and changes of address should be sent to Pacific Journal of Mathematics, 103 Highland Boulevard, Berkeley, California, 94708.

PUBLISHED BY PACIFIC JOURNAL OF MATHEMATICS, A NON-PROFIT CORPORATION

Printed at Kokusai Bunken Insatsusha (International Academic Printing Co., Ltd.), 7-17, Fujimi 2-chome, Chiyoda-ku, Tokyo, Japan.

* Acting Managing Editor. 


\section{Pacific Journal of Mathematics}

\section{Vol. 32, No. 3 \\ March, 1970}

Shair Ahmad, Dynamical systems of characteristic $0^{+} \ldots \ldots \ldots \ldots \ldots$

Charles A. Akemann and Bernard Russo, Geometry of the unit sphere of a $C^{*}$-algebra and its dual............................ 575

Philip Bacon, The compactness of countably compact spaces ......... 587

Richard Blaine Barrar and Henry Loeb, On the continuity of the nonlinear Tschebyscheff operator ............................ 593

L. Carlitz, Factorization of a special polynomial over a finite field ....... 603

Joe Ebeling Cude, Compact integral domains .................... 615

Frank Rimi DeMeyer, On automorphisms of separable algebras. II . . . . . 621

James B. Derr, Generalized Sylow tower groups .................. 633

Raouf Doss, Some inclusions in multipliers ................... 643

Mary Rodriguez Embry, The numerical range of an operator........... 647

John Froese, Domain-perturbed problems for ordinary linear differential

operators..................................... 651

Zdeněk Frolík, Absolute Borel and Souslin sets ..................... 663

Ronald Owen Fulp, Tensor and torsion products of semigroups .......... 685

George Grätzer and J. Płonka, On the number of polynomials of an

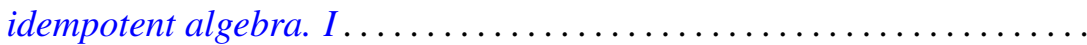

Newcomb Greenleaf and Walter Read, Positive holomorphic differentials on

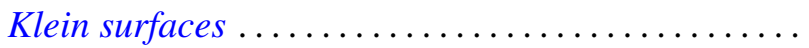

John Willard Heidel, Uniqueness, continuation, and nonoscillation for a second order nonlinear differential equation ................. 715

Leon A. Henkin, Extending Boolean operations................... 723

R. Hirshon, On hopfian groups .......................... 753

Melvin Hochster, Totally integrally closed rings and extremal spaces ..... 767

R. Mohanty and B. K. Ray, On the convergence of a trigonometric integral ..................................

Michael Rich, On a class of nodal algebras .................... 787

Emile B. Roth, Conjugate space representations of Banach spaces ........ 793

Rolf Schneider, On the projections of a convex polytope .............. 799

Bertram Manuel Schreiber, On the coset ring and strong Ditkin sets ...... 805

Edgar Lee Stout, Some remarks on varieties in polydiscs and bounded

holomorphic functions .........................

James Edward Ward, Two-groups and Jordan algebras . 